\title{
Dependence structure in the Australian electricity markets: new evidence from regular vine copulae
}

Article

Accepted Version

Creative Commons: Attribution-Noncommercial-No Derivative Works 4.0

Apergis, N., Gozgor, G., Lau, C. K. M. and Wang, S. (2020) Dependence structure in the Australian electricity markets: new evidence from regular vine copulae. Energy Economics, 90. 104834. ISSN 0140-9883 doi:

https://doi.org/10.1016/j.eneco.2020.104834 Available at https://centaur.reading.ac.uk/91246/

It is advisable to refer to the publisher's version if you intend to cite from the work. See Guidance on citing.

To link to this article DOI: http://dx.doi.org/10.1016/j.eneco.2020.104834

Publisher: Elsevier

All outputs in CentAUR are protected by Intellectual Property Rights law, including copyright law. Copyright and IPR is retained by the creators or other copyright holders. Terms and conditions for use of this material are defined in the End User Agreement.

www.reading.ac.uk/centaur 
Central Archive at the University of Reading

Reading's research outputs online 


\title{
Dependence Structure in the Australian Electricity Markets: \\ New Evidence from Regular Vine Copulae
}

\begin{abstract}
In this study, regular vine copula was used to investigate the dependence structure of electricity prices at the state level in the Australian National Electricity Market (NEM), during three periods related to the adoption and abolition of the carbon tax. In the pre-carbon period, we found evidence of tail dependence separately in the northern and southern NEM, but not across them. During the carbon period, the joint spike in the northern NEM disappeared, and the tail dependence in the southern NEM decreased. In the post-carbon period, the best dependence structure turned out to be a flexible structure of the regular vine, which exactly matches the geographical infrastructure connectedness of transmission wires. Besides, both upper and lower tail dependences were found in all adjacent states after the abolition of the carbon tax, suggesting a more integrated market regarding tail dependence. Our findings have substantial implications for risk management in the NEM, especially for those participants exposed to multiple states.
\end{abstract}

Keywords: Australian National Electricity Market; dependence structure; tail dependence; Rvine copula.

JEL Classification Codes: Q41; Q47; C32; C46. 


\section{Introduction}

As in the case of non-storable energy markets, electricity markets are characterized by particular features that make them distinctive from other energy markets. Among the different electricity markets in the world, the Australian National Electricity Market (NEM) has a unique structure. This is because most of the electricity in Australia is generated, purchased, and sold in free markets, requiring the market to match the supply and demand for electricity simultaneously. This task is performed by NEM for the east coast and southern states. According to Manner (2019), NEM conducts the most efficient auction of electricity in the world. However, the market prices of power in the spot NEM are incredibly volatile, and there is an asymmetric and nonlinear price dependency among markets in different states. Specifically, in the short-term, prices can fluctuate to levels immensely more substantial than the long-term (average daily) price. Consequently, market participants (retailers and generators) and traders need efficient risk management instruments when transacting in the NEM. At this stage, to reduce price volatility, market participants generally make contracts for hedging purposes, such as fixing the future electricity price. These derivative contracts can be informally and formally traded in over-the-counter markets and the Australian Securities Exchange (ASX), respectively. The ASX has eight futures products, which are hinged on "base-load," "peak-period," electricity purchases, and sales in each quarter for four states, i.e., New South Wales, Queensland, South Australia, and Victoria. Therefore, it is essential to provide an efficient risk management method for market participants in the NEM.

To this end, the present study aimed at analyzing the dependence structure of electricity markets across Australian states by using the vine copula methodological approach. Specifically, the analysis explored the dependence structure among the daily electricity prices in five Australian states, spanning the period July 1, 2008, to June 30, 2019. The copula approach is a powerful tool used to explore the dependence structure across markets (Nelsen, 
1999). In the multivariate case, vine copula can provide an efficient risk management approach, particularly for studying tail dependence among the daily electricity prices in different states (Sukcharoen and Leatham, 2017). Among specific variants of the vine copula approaches, the regular (R-) vine copula method stands out because of its distinctive advantages. Compared with the canonical (C-) vine and drawable (D-) vine copulas, the R-vine copula has a substantially flexible structure, which can help researchers find a more intrinsic dependence structure.

In recent literature, copula approaches have been extensively used in energy markets. A strand of the literature has used copula approaches within the energy and oil markets (Czado et al., 2010; Reboredo, 2011; Sukcharoen and Leatham, 2017). Certain studies have also considered the copula approach and analyzed the dependence between energy (mainly oil markets) and stock markets (Aloui et al., 2013a; Li and Wei, 2018; Reboredo, 2015; Sukcharoen et al., 2014; Wen et al., 2012). Another strand of the literature has focused on the dependence between energy markets and exchange rates (Aloui et al., 2013b; Reboredo et al., 2014). These studies provide evidence that the copula approach is robust to the frequency of data used, but it can model the presence of asymmetric effects across different markets. The most closely related article to this study is Manner et al. (2019), which focused on forecasting the joint distribution of Australian electricity prices by a stochastic autoregressive D-vine copula.

In agreement with these studies using various copula approaches, this paper investigated the dependence structure across electricity markets in Australian states by a more flexible vine copula structure, the R-vine copula. This study sheds further light on the critical changes in the dependence structures of three periods (pre-carbon, carbon-tax, and post-carbon) to provide implications for risk management in the NEM. To the best of our knowledge, this is the first paper in the literature to examine the dependence structure across electricity markets 
by the R-vine copula. Our contribution is twofold; first, we revealed the dependence structure across electricity markets in Australian states in three periods. In the pre-carbon period, we found evidence of tail dependence separately in the northern and southern NEM, but not across them. During the carbon period, the joint spike in the northern NEM disappeared, and the tail dependence in the southern NEM decreased. We found that the dependence structure in the post-carbon period matched the geographical presence of the national electricity transmission grid, which indicated that the structure could only be revealed by R-vine copula. Both upper and lower tail dependences were found in all adjacent states after the abolition of the carbon tax, suggesting a more integrated market concerning tail dependence. Second, our approach offers a risk-management tool based on the R-vine copula and valuable insights into the tail risks of unfavorable price movements for market participants in the Australian NEM, especially for participants involved in multiple states.

The remaining parts of this paper are organized as follows. Section 2 presents a brief review of related literature. Section 3 provides the methodology, while Section 4 reports the data and descriptive statistics. Section 5 presents the empirical results, Section 6 discusses our findings in-depth, and Section 7 concludes.

\section{Literature Review}

An increasing number of studies have used the copula methodological approach to examine the presence of dependence structure across markets, including energy markets. It is also imperatively essential to note that there are a few numbers of papers that analyzed the price dynamics in the NEM. Hu et al. (2005) demonstrated strategic bidding and rebidding behavior in the NEM, spanning the period May 1, 2002, to May 31, 2003. They indicated that market prices are mainly controlled by the larger generators, by providing 'capacity' quotations rather than 'price' quotations. Additionally, they highlighted that larger generators do not reduce the 
market price during peak periods by exploiting the presence of market regulations, which leads to inefficient markets, particularly during peak periods. Higgs (2009) indicated that the introduction of the Queensland-New South Wales Interconnector (QNI) is the central issue of the significant interaction between both states. It is also noteworthy that although the findings emphasize critical interconnections between related pairs, the author concluded that the Australian electricity markets have limited interdependence across the country. This evidence implies that at the state level, the Australian electricity markets are characterized as isolated markets from January 1, 1999, to December 31, 2007. Cutler et al. (2011) used 30-min spot electricity price data in South Australia, spanning the period September 2008 to August 2010, to examine market dynamics. In determining electricity prices in South Australia, it was demonstrated that the demand side of the market is a more critical factor than the supply side. According to their results on the negative correlation between spot electricity price and wind generation, wind power is a significant driver of electricity prices. However, according to Bell et al. (2017), there is no considerable causality between electricity price and wind generation in South Australia, since wind power outputs are associated with the events of extreme amounts. These findings imply a significant role in the demand-side dynamics (i.e., wind generation) in spot electricity prices at the state level. However, Cutler et al. (2011) only examined the case of South Australia; and therefore, any price interactions across more states were neglected.

Analyzing the price interactions across states in the NEM is a relatively noteworthy research task. Considering the intraday 5-min electricity price data from December 8, 1998, to May 5, 2016, Apergis et al. (2017a) examined volatility spillover across four Australian states: New South Wales, Queensland, South Australia, and Victoria. Their analysis focused on the asymmetries in electricity price volatility, known as 'good' volatility and 'bad' volatility. They found that electricity markets in these states have interacted asymmetrically, and the presence of some degree of market power has been utilized by generators across related electricity 
markets. Apergis et al. (2017b) obtained significant convergence at wholesale electricity prices in New South Wales, Queensland, and Victoria. Still, there was no significant evidence in the case of South Australia, implying that there are common characteristics of electricity markets in these states. Their findings indicated that similar carbon tax regimes could explain the common features in these states.

Several studies have used vine copula approaches in energy variables and markets. Sukcharoen and Leatham (2017) applied the C-vine and D-vine copula models for price margins between refined products (gasoline and heating oil) and the production cost of crude oil. The authors concluded that the D-vine copula approach is more suitable for hedging purposes when it comes down to related assets in the refinery. Using the time-varying Gaussian copula approach, Pircalabu et al. (2017) investigated the tail dependence between wind power production and electricity prices in Denmark. They found that the risk is underestimated if independence is assumed between prices and wind power production. They further proposed to reduce the risk by using an underlying hedging instrument in the forward market. Finally, Manner et al. (2019) used the stochastic autoregressive D-vine (SCAR-Dvine) copula to forecast the out-of-sample log of daily electricity prices in the Australian NEM, during 20102015. Based on their findings, the SCAR-Dvine copula outperformed various other types of approaches, because it provided a robust back-testing procedure. The authors concluded that the SCAR-Dvine approach could be considered as a hedging tool for market participants (especially retailers) in the NEM.

Overall, there is still a gap in the empirical literature for understanding the dependence structure across spot electricity prices in the case of the NEM due to two aspects. First, although D-vine copula was used by Manner et al. (2019) for forecasting, such vine copula is limited by the drawable structure. It is incapable of reflecting a more general dependence structure. Second, the existing literature typically partitions the data into different periods in an arbitrary 
manner. For forecasting evaluation, Manner et al. (2019) split the data into the in-sample period (2010-2012) and the out-of-sample period (2013-2015). However, structural breaks caused by policy changes affect electricity prices in Australian states and their dependence structure, such as the adoption and abolition of the carbon tax from July 2012 and June 2014. Using daily data, spanning the period July 1, 2008, to June 30, 2019, this paper employed R-vine copula models to investigate the dependence structure of the NEM in three different periods, pre-carbon (2008-2012), carbon tax (2012-2014), and post-carbon (2014-2019). This paper revealed the evolvement of dependence structure across states while generating substantial implications for risk management in the NEM. Accurately, the findings show that specific policy changes in the NEM are related to changes in the dependence structure across the Australian states.

\section{Methodology}

Since electricity cannot be stored on a massive scale at a low-cost, electricity price is more relevant for market participants, rather than the (log) returns in financial studies. Traditionally, a natural logarithm of the spot price is taken on electricity prices to scale down the numeric values (e.g., Lucia and Schwart, 2002; Manner et al., 2019). However, this is only applicable for prices that cannot take negative values. Electricity prices at lower frequencies, such as weekly or monthly data, tend to be strictly positive. Still, the aggregation removes a lot of the tail behavior that is particularly interesting in this study. Following Pircalabu et al. (2017), we decided to use the original price without logarithms, because daily electricity prices in NEM can take negative values, as shown in Section 4.1. Based on the choice of the variable of interest, we started Section 3.1 by decomposing electricity prices into deterministic and stochastic components. Then, we elaborated on how to model the stochastic components through the $\operatorname{ARMA}(p, q)-\operatorname{GARCH}(1,1)$ model, which produces standardized residuals for copula analysis. We illustrated two preliminary dependence analysis tools, quantile dependencies, and a 
structural break test. Finally, an explanation is presented of our primary method of modeling the multidimensional dependence structure by R-vine copula.

\subsection{Deterministic and Stochastic Components}

Following Huisman and Mahieu (2003) and Manner et al. (2019), the spot price for electricity in State $i$ at time $t$ consists of a deterministic component $f_{i, t}$ and a stochastic part $x_{i, t}$ :

$$
p_{i, t}=f_{i, t}+x_{i, t} \quad i=1, \ldots, 5
$$

where the component $f_{i, t}$ is a deterministic function of time that captures predictable patterns, such as seasonality, trend, and special events. The remaining component $x_{i, t}$ follows a stochastic process involving autocorrelation and heteroskedasticity, which has been modeled in Section 3.2. Considering the characteristics of the NEM, we decided to specify the deterministic component $f_{i, t}$ as:

$$
f_{i, t}=\beta_{i, 0}+\boldsymbol{\beta}_{\boldsymbol{i}, \mathbf{1}} \boldsymbol{D}_{t}^{(\text {Year })}+\boldsymbol{\beta}_{\boldsymbol{i}, \mathbf{2}} \boldsymbol{D}_{t}^{(\text {Month })}+\boldsymbol{\beta}_{\boldsymbol{i}, \mathbf{3}} \boldsymbol{D}_{t}^{(\text {DoW })}+\boldsymbol{\beta}_{\boldsymbol{i}, \mathbf{4}} \boldsymbol{D}_{\boldsymbol{i}, t}^{(\text {Event })}
$$

where $\beta_{i, 0}$ is the average price level at each state. $\boldsymbol{D}_{t}^{(\text {Year })}$ is a set of dummy variables indicating the fiscal year in the NEM, which is between every July in different years. We decided to use the fiscal year rather than the calendar year, because the market price cap (MPC), the maximum price that generators can bid, is adjusted ${ }^{1}$ on July 1 based on movements in the consumer price index (Simshauser, 2019). $\boldsymbol{D}_{t}^{(\text {Month) }}$ is a group of dummy variables reflecting annual seasonality. $\boldsymbol{D}_{t}^{(D o W)}$ is a collection of dummy variables incorporating the day-of-week effect. Lastly, $\boldsymbol{D}_{\boldsymbol{i}, t}^{(\text {Event })}$ is the dummy variable denoting a special event in a specific state. This

\footnotetext{
${ }^{1}$ MPC was $\$ 14,500 / \mathrm{MWh}$ in $2018 / 19, \$ 14,200 / \mathrm{MWh}$ in $2017 / 18$, $\$ 14,000 / \mathrm{MWh}$ in $2016 / 17, \$ 13,800 / \mathrm{MWh}$ in 2015/16, $\$ 13,500 / \mathrm{MWh}$ in 2014/15, and $\$ 13,100 / \mathrm{MWh}$ in 2013/14.
} 
study only considered the Basslink outage ${ }^{2}$ between December 2015 and April 2016, which caused the energy crisis in Tasmania.

\subsection{Marginal Models for Stochastic Components}

Copula-based models enable the separation of marginal behavior and dependence structure across markets; this advantage enhances the efficiency of model estimations (Patton, 2012). After removing the deterministic component, we focused on modeling the stochastic element featured by autocorrelation and heteroskedasticity. To perform this task, the $\operatorname{ARMA}(p, q)$ GARCH(1,1) model ${ }^{3}$ with skewed $t$ innovation was selected due to three motivations. First, the level equation is set to be an $\operatorname{ARMA}(p, q)$ to capture the autocorrelation. Second, the volatility equation is described by a $\operatorname{GARCH}(1,1)$ to reflect the heteroskedasticity. Third, we observed many outliers in the data, especially for right tail outliers due to the definite price spikes. Thus, the standardized residuals are chosen to follow the simple and flexible skewed $t$ distribution of Hansen (1994). The marginal model ${ }^{4}$ is presented below:

$$
\begin{aligned}
& x_{i, t}=\sum_{k=1}^{p} \phi_{i, k} x_{i, t-k}+\varepsilon_{i, t}+\sum_{s=1}^{q} \theta_{i, s} \varepsilon_{i, t-s} \quad i=1, \ldots, n \\
& \sigma_{i, t}^{2}=\omega_{i}+\alpha_{i} \varepsilon_{i, t-1}^{2}+\beta_{i} \sigma_{i, t-1}^{2} \\
& \varepsilon_{i, t}=\sigma_{i, t} \eta_{i, t}, \quad \eta_{i, t} \mid \Omega_{i, t-1} \sim \text { i.i.d. skewed } t
\end{aligned}
$$

where $x_{i, t}$ is the stochastic component taken from Equation (1), $\sigma_{i, t}$ is the conditional volatility, $\Omega_{i, t-1}$ is the information set generated by $\left(x_{i, t-1}, x_{i, t-2}, \ldots\right)$, and $\eta_{i, t}$ is the standardized residuals, which is in line with the skewed $t$ distribution regarding shape and skewness parameters. The quasi-maximum likelihood method (Bollerslev and Wooldridge,

\footnotetext{
${ }^{2}$ For details, refer to https://en.wikipedia.org/wiki/2016_Tasmanian_energy_crisis (Accessed on November 23, 2019).

${ }^{3}$ Higher orders in the GARCH part of the ARMA-GARCH model, such as ARMA(p,q)-GARCH(2,2), have also been experimented and show the fallacies that they cannot outperform the ARMA(p,q)-GARCH( 1,1$)$ model in terms of BIC.

${ }^{4}$ There is no intercept in the level equation because removing the deterministic component results in having a stochastic component always with zero means.
} 
1992) is applied to estimate the marginal models, and the best lag lengths of $\operatorname{ARMA}(p, q)$ $\operatorname{GARCH}(1,1)$ are selected by the BIC information criteria.

Specifying the correct marginal models is a crucial step. Without correctly specified marginal models, the estimated copula model would be unable to capture the correct dependence structure. This is because the copula model has the i.i.d. assumption on the observations. To ensure that the standardized residuals are close to the i.i.d. assumption (at least no autocorrelation and no heteroskedasticity), we considered three diagnostic tests developed by Fisher and Gallagher (2012). The weighted Ljung-Box analysis is applied to standardized residuals to examine the hypothesis of no serial correlation. To check the hypothesis of no heteroskedasticity, the weighted Ljung-Box test is also used on squared standardized residuals, and the weighted ARCH-LM test is employed on standardized residuals.

Before dependence analysis in the copula framework, the standardized residuals must be transformed into the (conditional) probability integral transform variables, $U_{i, t}=$ $F_{i}\left(x_{i, t} \mid \Omega_{i, t-1}\right)$, which are uniformly distributed in the domain $[0,1]$. The probability integral transform (PIT) can be implemented in both parametric and nonparametric ways (Patton, 2013). In this study, according to Dißmann et al. (2013), we employed the empirical PIT because there is always some uncertainty in the residual distribution if the sample size is large. To be specific, the empirical PIT is conducted via the empirical distribution function (EDF):

$$
\hat{F}_{i}(\varepsilon) \equiv \frac{1}{T+1} \sum_{t=1}^{T} \mathbb{1}\left(\hat{\varepsilon}_{i, t} \leq \varepsilon\right)
$$

where $\mathbb{1}(\cdot)$ is the identity function.

\subsection{Quantile Dependence}

As a preliminary analysis before vine copula modeling, quantile dependence can be used as a convenient tool to visualize the pairwise dependence structure in the lower and upper joints of their support. For positively dependent variables (our case), it is defined as: 


$$
\lambda^{q}= \begin{cases}\mathbb{P}\left(U_{1, t} \leq q \mid U_{2, t} \leq q\right), & 0<q \leq 1 / 2 \\ \mathbb{P}\left(U_{1, t}>q \mid U_{2, t}>q\right), & 1 / 2<q<1\end{cases}
$$

And it can be estimated by finite samples

$$
\hat{\lambda}^{q}= \begin{cases}\frac{1}{T q} \sum_{t=1}^{T} \mathbb{1}\left(U_{1, t} \leq q \mid U_{2, t} \leq q\right), & 0<q \leq 1 / 2 \\ \frac{1}{T q} \sum_{t=1}^{T} \mathbb{1}\left(U_{1, t}>q \mid U_{2, t}>q\right), & 1 / 2<q<1\end{cases}
$$

When compared with a scalar dependence measure, such as Pearson's correlation, Kendall's tau, and Spearman's rho, quantile dependence provides a more valuable description of the pairwise dependence structure. This is because it shows the full picture of the dependence strength, as we move from the center $(q=1 / 2)$ to the two tails. Additionally, it reveals whether the dependence structure is symmetric in the lower $(0<q \leq 1 / 2)$ and upper $(1 / 2<q<1)$ supports. The preliminary analysis of quantile dependence offers insights on the choice of possible parametric copulas, which should capture the empirical features observed in the empirical data.

\subsection{Structural Breaks in Dependence}

Since our data covers an extended period, it is unrealistic to assume that the dependence structure remains constant all the time. In the literature, there are two methods of dealing with such a problem. The first method assumes that the dependence is intrinsically time-varying and follows some functional forms, including the Generalized Autoregressive Score (GAS) model (Creal et al., 2012; Pircalabu et al., 2017), stochastic autoregressive copula (SCAR) method (Manner et al., 2019) and the regime-switching approach (Wang et al., 2013). The second method considers that the dependence is subject to structural breaks, due to external shocks, such as policy changes. In this paper, we took the position of the second method and considered structural breaks. Patton (2013) provided the details of two structural break tests (a simple test and a sup test) in a rank correlation, depending on whether the break date is known or unknown. 
The present study used a test based on Patton's (2013) simple test, which assumes that the researchers have prior knowledge about the time when a break in the dependence may have occurred. The justification for using the simple test is that we know the exact dates when the Australian government adopted and abolished the carbon pricing scheme, which is discussed in detail in Section 4.4. It should be highlighted that we are more interested in the Kendell's tau, rather than the Spearman's rho used in Patton (2013) because the former is required in choosing the best tree structure for vine copulas, which is elaborated in Section 3.4. The hypothesis test can be formulated in the following way:

$$
\begin{aligned}
& H_{0}: \tau_{1}=\tau_{2} \quad \text { v.s. } H_{A}: \tau_{1} \neq \tau_{2} \\
& \tau_{t}= \begin{cases}\tau_{1}, & t \leq t^{*} \\
\tau_{2}, & t>t^{*}\end{cases}
\end{aligned}
$$

where $t^{*}$ is the break date determined by the prior knowledge. The test statistics is $\left|\hat{\tau}_{1}-\hat{\tau}_{2}\right|$, where $\hat{\tau}_{1}$ and $\hat{\tau}_{2}$ are estimated by the sample before and after the break.

With justification from Chen and Fan (2006) and under the null hypothesis that the dependence measure is equal before and after $t^{*}$, it turns out that the $p$-value of the statistics can be obtained via the simple i.i.d. bootstrap procedure: 1) generate the bootstrapped standardized residuals by randomly drawing rows with replacement from the original matrix of standardized residuals; 2) estimate the $\hat{\tau}_{1}^{*}$ and $\hat{\tau}_{2}^{*}$ and calculate the bootstrapped statistics; 3 ) repeat Steps 1 and 2 a large number of times; 4) obtain the $p$-value by calculating the frequency that the original test statistics are less than the bootstrapped ones. Since we directly bootstrapped the standardized residuals rather than the original data, no marginal model reestimation was involved, and thus this simple i.i.d. bootstrap procedure can quickly be completed in practice. 


\subsection{Vine Copula Method}

Copula models can capture the complex and nonlinear dependence structure of a multivariate distribution across markets. Therefore, copula methods are superior to traditional approaches, such as Vector Autoregressive (VAR) causality models, because they are only capable of capturing a linear-dependence relationship, but not the asymmetric distributional dependence across markets. The copula model used in the analysis provides the opportunity to explore both the tail dependence and the asymmetric dependence across different electricity markets. ${ }^{5}$

Among the different variations of copula models, the approach of vine copula initially introduced by Joe (1996), is widely used to model high dimensional dependence. Its core idea is to decompose the multivariate copula density function into individual pairwise copula density functions. Thus, the multidimensional dependence structure can be flexibly modeled by pair copula construction (PCC). The PCC method was developed by Bedford and Cooke (2001, 2002) and Kurowicka and Cooke (2006). Aas et al. (2009) used a maximum pseudolikelihood approach to develop an inference method for pair-copula decomposition, and the vine copula concept was popularized by their empirical demonstration, using non-Gaussian pair copulas on financial data. Recent surveys on vine copula were conducted by Czado (2010), Czado et al. (2010), and Joe and Kurowicka (2011).

A parametric vine copula consists of three components: 1) a set of linked trees to specify the pairs of variables and their conditioning variables; 2) the copula family for each pairwise link; 3) the parameters in each pairwise copula. Generally, there are three types of vine copulas, namely $\mathrm{C}$-vine, D-vine, and R-vine, depending on how the nodes and edges in the tree of a vine copula are connected. The C-vine copula is in a "star" structure with one central node connecting with all other nodes in all levels of trees, which is suitable for a case where there is

\footnotetext{
${ }^{5}$ The modeling of both the left tail and the right tail dependence among electricity markets can also measure the probability of simultaneous extreme events due to extreme weather conditions and price shocks in the global coal markets.
} 
a dominating variable affecting others. D-vine copula has a "path" structure, and no node is connected to more than two other nodes in each tree. Both C-vine and D-vine are sub-classes of a general class named R-vine, which has a flexible structure such that the nodes in each tree are connected through the edges, and each node comes from a specific edge in the previous tree. The only restriction in R-vine construction is the proximity condition that two nodes in each tree are connected by an edge if they share a common node in the previous tree. The general density formula for R-vine copula specification is expressed as:

$$
f_{1, \ldots, n}(\boldsymbol{x})=\prod_{k=1}^{n} f_{k}\left(x_{k}\right) \prod_{i=1}^{n-1} \prod_{e \in E_{i}} C_{C_{e, a}, C_{e, b \mid D_{e}}}\left(F_{C_{e, a} \mid D_{e}}\left(x_{C_{e}, a} \mid \boldsymbol{x}_{D_{e}}\right), F_{C_{e, b} \mid D_{e}}\left(x_{C_{e}, b} \mid \boldsymbol{x}_{D_{e}}\right)\right)
$$

where $\boldsymbol{F}=\left(F_{1}, \ldots, F_{n}\right)$ is a vector of continuous invertible distribution functions, $f_{i}$ is the density of $F_{i}, \boldsymbol{x}=\left(x_{1}, \ldots, x_{n}\right)$, the edge $e=\{a, b\}, E_{i}$ stands for the edge set in the $i$ th tree, and $\boldsymbol{x}_{D_{e}}$ denotes the conditional variables that are in $D_{e}$. The density for C-vine and D-vine can be written in a more simplified way (Aas et al., 2009).

As a result of the restriction in the PCC of C-vine and D-vine, their estimation can be facilitated by the maximum likelihood method. The consequence of the loose restriction in Rvine is an enormous number of possible R-vine tree structures, and the maximum likelihood method is often infeasible and is implementable if the dimension is large. To tackle this problem, Dißmann et al. (2013) developed a sequential and heuristic approach for the R-vine copula inference, which is summarized below. The full R-vine copula specification can be divided into three separate tasks:

a) The determination of an appropriate R-vine tree structure.

b) The choice of a bivariate copula family for each pair.

c) The estimation of parameters for each bivariate copula.

To fulfill task $a$ ), the principle is to select the structure of the first spanning tree that can maximize the sum of absolute empirical Kendall's taus, 


$$
\max \sum_{e=\{i, k\} \text { in spanning tree }}\left|\hat{\tau}_{j, k}\right|
$$

For trees at a higher level, the maximization concerns all conditional variable pairs $\{j, k \mid D\}$. Regarding Task $b$ ), the best bivariate copula family is selected by the information criteria, AIC. In this study, the following copula families were considered: Gaussian, Student's t, Clayton, Gumbel, Frank, Joe, and their survival counterparts. The independent copula is used if the independence test of Genest and Favre (2007) cannot be rejected. Regarding Task c), the bivariate copula parameters are estimated by the maximum likelihood method. In this study, our implementation was based on an R package "Vine Copula" (Nagler et al., 2019).

\section{Data}

The analysis utilized the daily average spot price $(\$ / \mathrm{MWh})$ per region, spanning the period July 1, 2008, to June 30, 2019. The sample consisted of five states: Queensland (QLD), New South Wales (NSW), Victoria (VIC), South Australia (SA), and Tasmania (TAS). Our data was downloaded from the AEMO website ${ }^{6}$. The date of commencement was chosen because the Snowy region ${ }^{7}$ was eliminated as a region on July 1, 2008, and was divided into NSW and VIC. During our sample period, there were 4017 observations in each state.

We decided to use the daily frequency, rather than weekly or monthly frequencies because aggregation removes a lot of the tail behavior that may be particularly interesting to market participants. Since a large scale of electricity cannot be stored at a low-cost, the dependence between the prices would be much more relevant for market participants, but not the $(\log )$ returns typically used in financial studies. Unlike some literature that may take the

\footnotetext{
${ }^{6}$ https://www.aemo.com.au/Electricity/National-Electricity-Market-NEM/Data-dashboard\#aggregated-data. ${ }^{7}$ For details, visit https://en.wikipedia.org/wiki/Snowy Mountains_Scheme (Accessed on November 13, 2019).
} 
logarithm of the electricity price to scale down, we decided to use the original price without logarithm because the prices in NEM could go negative.

Figure 1 shows the time series plot of electricity prices in the five states. As can be observed, the spot price has high volatility, and among the most paramount features are large spikes, which can shoot up to several dozen times the average price for short periods. The spikes can be explained by the rigid demand curve of electricity (Higgs and Worthington, 2008). Another noticeable feature is that the spot price can even go contrary. The National Electricity Rules set a minimum spot price at $-1,000$ per $\mathrm{MWh}$ (market floor price). The economic rationale behind the adverse market floor price is mainly to allow generators to stay online when the cost of shutting down and restarting their plants could be higher than the cost of staying online.

Additionally, renewable generators may cost less if they choose to stay online because they have support mechanisms, such as the Renewable Energy Target Scheme. It is worthwhile to point out the abnormal price in TAS from December 2015 to April 2016, in which the Basslink (the only interconnector between VIC and TAS) experienced a significant outage and caused an energy crisis in TAS. The price increased steadily and did not return to the average level until the first stage of the repairs was completed in April 2016.

Table 1 presents the descriptive statistics of the spot price in the five states. Firstly, we placed focus on the first four moments. Except for SA, there was no significant difference in the mean level across the other four states. Additionally, volatility (measured by the standard deviation) in SA was prominently higher compared to the other states. Due to the existence of large spikes, the distributions of prices in five regions were all right-skewed and had substantially high kurtosis. Secondly, we analyzed the summative distribution information. Apart from NSW, the minimum price in the other four states can go negative. The maximum amount caused by the spikes can be indeed several dozen times higher than its median value. 
Even for the $90 \%$ quantile values, they are still at a reasonable level, approximately two times higher than the median level. This evidence has encouraged us to report the frequency of price anomalies. We defined the defect of positive spikes as the price more substantial than the quantity of the local mean plus three times the local standard deviation. ${ }^{8}$ The anomaly of negative prices is the price of less than zero. According to our definition, it can be observed that spikes empirically occur around $1 \%$ frequency, but negative prices rarely take place. Thirdly, we present the estimated coefficients for both Pearson's linear correlation (above the main diagonal) and Kendall's tau (below the main diagonal) in Table 2. Although Pearson's linear correlation showed slight or medium linear dependence, Kendall's tau, as a rank correlation, indicated a specific strength of dependency in the electricity prices across five states. For example, the linear correlation between VIC and QLD was only 0.11, while Kendall's tau was 0.62 . The evidence here implies the existence of a nonlinear relationship and provides strong motivation for using the copula method to capture such a non-trivial dependence structure.

\section{Empirical Results on the Dependence Structure}

\subsection{Results from Marginal Models}

After removing the deterministic component (estimated results are presented in Table A1 of Appendix A), we used $\operatorname{ARMA}(p, q)-\operatorname{GARCH}(1,1)$ to model the stochastic part of the electricity price. The lag length was capped at seven, i.e., $p, q \leq 7$, and the best lag length was selected by BIC. Table 3 presents the estimated parameters for the marginal models ${ }^{9}$. For instance, a relatively large model, $\operatorname{ARMA}(7,2)-\operatorname{GARCH}(1,1)$, was chosen for NSW, while the best model

\footnotetext{
${ }^{8}$ Local mean and local standard deviation at time $t$ are calculated based on the range $[\min (0, t-182), \max (t+$ $182, T)$ ], i.e., a centralized window minus or plus 182 calendar days (truncated at 0 and $T$ ).

${ }^{9}$ We noted that the estimated alpha and beta in the GARCH equation might have their summation close to one. This evidence is due to the substantial volatility caused by positive spikes. Thus, we have also tried the IGARCH model, which is a strictly stationary process without the second moment. Still, we found little difference in the results of the marginal model and the subsequent analysis.
} 
of QLD was a parsimonious model of $\operatorname{ARMA}(2,1)-\operatorname{GARCH}(1,1)$. The estimated shape and skewness parameters of the error distribution reflect the right-skewed and heavy-tail features revealed in Section 4.2.

The crucial issue in this step of marginal modeling is the residuals diagnostics because the copula analysis requires i.i.d. observations. If the standardized residuals are subject to autocorrelation and heteroskedasticity, then the conclusion from the copula analysis may not be valid. In this study, three weighted diagnostic tests developed by Fisher and Gallagher (2012) were applied, and Table 4 presents their $p$-values. In our case, the standardized residuals from the chosen marginal models of all five states cannot reject the null hypothesis at 5\% significance level; thus, it exhibited no serial autocorrelation and heteroskedasticity.

\subsection{Results from Quantile Dependence}

After verifying the i.i.d. assumption, the empirical PIT described in Equation (4) was used to obtain the PIT variables in the domain [0,1]. Figure 2 shows the pairwise scatter plots of the PIT variables and their Kendall's tau. After the ARMA-GARCH filtering, it was found that Kendall's tau of standardized residuals are less than those of the original data.

As a preliminary exploration of the dependence structure, we computed the quantile dependence, based on Equation (6) with $q \in[0.025,0.975]$, for all ten possible pairs of states, and the results are presented in Figure 3. It can be observed that most pairs have symmetric dependence structures. When moving from the center $(q=0.5)$ to the left tail $(q$ near 0$)$ or right tail ( $q$ near 1), there is a mixing conclusion on which tail has a larger dependence, but their differences are not noticeable. ${ }^{10}$

\footnotetext{
${ }^{10}$ The time series stationary bootstrap procedure is explained in detail by Patton (2013). This method can be implemented to show the confidence interval of the difference in quantile dependence. Again, due to space constraints, we decided not to report the difference in quantile dependence.
} 


\subsection{Results from Structural Breaks in Dependence}

In 2011, the Gillard Labor Government introduced the carbon pricing scheme ${ }^{11}$ in Australia, commonly known as the carbon tax, with a legislation named Clean Energy 2011, which took effect on July 1, 2012. Although there was a decline in carbon emissions after the imposition of the carbon tax, the net result was a deadweight cost to the economy because it indirectly increased electricity costs for households and industries (Apergis et al., 2019). The Abbott Government repealed the carbon tax on July 1, 2014, and replaced it with the Emission Reduction Fund.

Apergis et al. (2019) showed that carbon tax is associated with common market characteristics across different states because of "a greater degree of interconnectedness via greater transfer capacity on interstate interconnectors, linking QLD, NSW, and VIC relative to the interconnectors linking TAS and SA to VIC." We suspected that the dependence structure might also have structural breaks due to this policy change. Therefore, we considered the dates when the carbon tax was adopted and abolished as the known break dates and applied the structural break test described in Section 3.3.

Table 5 presents the $p$-values of the structural break test. We found strong evidence of structural breaks in the dependence of most state pairs on the dates of adopting and abolishing the carbon tax. Thus, the whole sample was divided into three periods: pre-carbon (Period 1: July 2008-June 2012), carbon tax (Period 2: July 2012 - June 2014), and post-carbon (Period 3: July 2014 - June 2019), and vine copula analysis was performed separately for the three periods.

\footnotetext{
${ }^{11}$ The carbon price was 23.00 Australian dollars per ton of emitted $\mathrm{CO}_{2}$ in the fiscal year 2012-13 and increased to 24.15 in the fiscal year 2013-14.
} 


\subsection{Results from Vine Copula}

Table 6 presents all results of the vine copula method ${ }^{12}$ described in Section 3.4 for the three periods, including the estimated parameters for the vine copula on the edges of all trees, as well as their corresponding Kendall's tau, lower tail dependence (LTD), and upper tail dependence (UTD). To more intuitively illustrate the dependence structure, Figure 4 displays Tree 1 of the best vine copula structure across all five states.

In the pre-carbon period, the best-selected tree structure was D-vine, according to the rule of maximizing the sum of absolute empirical Kendall's taus suggested by Dißmann et al. (2013). Based on this structure, we were able to derive several insightful observations. Firstly, the states in D-vine are connected in a way that is not necessarily based on the connectedness of their physical transmission lines in this period. In Tree 1, SA and VIC (in the southern NEM) are directly linked by the Student's $t$ copula (with Kendall's tau of 0.58), and the electricity grids in the two states are physically connected by the Heywood Interconnector ${ }^{13}$. Additionally, the networks of QLD and NSW (in the northern NEM) are directly connected by the $\mathrm{QNI}^{14}$, and their copula is in the Gumbel family (with Kendall's tau of 0.37 ), which only has UTD but no LTD. Other edges in Tree 1 are not backed by physical interconnectors. Secondly, it should be highlighted that the UTD in the pairs of SA/VIC and QLD/NSW are 0.52 and 0.46 , respectively. The high values of UTD indicate that both pairs tend to have positive spikes together. The other couples have either zero or minimal amounts of tail dependence. Thirdly, we observed that Kendall's taus in Trees 2-4 are close to zero. This evidence is consistent with

\footnotetext{
${ }^{12}$ The order of states in vine copula analysis is as follows: QLD, NSW, VIC, SA, and TAS. We observed that it is typical to have the simplifying assumption that copulas of conditional distributions do not depend on the values of the conditioning variables to keep the PCC method tractable for inference (Stöeber et al., 2013), and the simplifying assumption implies that the order of variables is irrelevant.

${ }^{13}$ The Heywood interconnector (275 KV) was commissioned in 1998 with the primary goal of moving low-cost coal-generated electricity from VIC to SA. Recently, it is also increasingly used to transmit wind-powered electricity from SA to VIC.

${ }^{14}$ The Queensland-New South Wales Interconnector (330 KV) started its services in 2001.
} 
Dißmann et al. (2013), believing that "the copula families specified in the first tree of the Rvine often have the greatest influence on the model fit." 15

In the carbon tax period, the best-selected tree structure remained the same as the Dvine. Besides, the pairwise Kendall's taus are close to the values in the previous period. The obvious observation is about the pair QLD/NSW. Its copula family changed from Gumbel to Frank, and thus the UTD shrank from 0.46 to 0 , which implies that these pairs tend to have much less joint positive spikes in the carbon tax period than before. Moreover, there was a slight decrease in the tail dependence of SA/VIC. Similar to the pre-carbon period, the higher trees play much fewer roles, with several independent copulae identified by the test of Genest and Favre (2007) in Trees 2 and 4.

In the post-carbon period, the best-selected tree structure becomes a general R-vine. There are three insightful observations. Firstly, the tree structure presented in Figure 4 (lower panel) exactly matches the geographical presence of the national electricity transmission lines across Australia (shown in Figure 5). This evidence implies that the dependence structure for the electricity markets reflects the infrastructure connectedness of transmission wires in the recent period. Secondly, we observed that the best copula in all pairs of adjacent states, namely NSW/QLD, VIC/NSW, VIC/SA, and TAS/VIC, is the Student's $t$ copula, which indicates the symmetric upper and lower tail dependences. This evidence implies the fact that they tend to have enormous positive/negative electricity prices simultaneously, which is a sign of more integrated NEM in terms of tail dependence, compared with the fact that most pairs have zero tail dependence in the carbon tax period. Thirdly, Tree 3 has two independent copulas, and we observed that the truncation test developed by Brechmann et al. (2012) could be used to determine whether the R-vine, in this case, be truncated at Tree 3 (i.e., setting all copulas in Trees 3 and 4 as independent copula).

\footnotetext{
${ }^{15}$ Note that D-vine is a subclass of R-vine.
} 


\subsection{Results from the Joint Probability in the Tails}

Since electricity cannot be stored, the Value-at-Risk (VaR), which is a risk measure typically calculated for financial returns, becomes less relevant in our context. From the perspective of retail buyers in NEM and the hedgers participating in the Australian Securities Exchange (ASX) energy contracts, their primary concern is whether the spot prices will simultaneously go to extremely high levels. Thus, we used the Monte Carlo simulation to investigate the joint probability of multiple states in upper tails. The repetition time was set as 1,000,000. For comparison, the multivariate Student's $t$ copula was used as the benchmark.

Table 7 presents the results of joint probability in the upper tails with quantiles more than $0.90,0.95$, and 0.99 for the empirical data, the vine copula, and the multivariate Student's $t$ copula, respectively. As can be observed, the joint probabilities generated by the vine copula method are close to the empirical likelihood. Thus, the framework based on the R-vine copula illustrates the accuracy of fitting the empirical joint probability of positive price spikes in multiple states. While the multivariate Student's $t$ copula can also produce a satisfactory result, it slightly deviates from the empirical ones when compared with the vine copula, with evidence of the average absolute difference ${ }^{16}$ of $0.38 \%$ in the multivariate Student's $t$ copula and $0.29 \%$ in the vine copula. Additionally, the vine copula has the advantage of being a parsimonious model with fewer parameters than the multivariate Student's $t$ copula. Overall, this model offers a risk-management tool based on the R-vine copula. At the same time, it provides valuable insights into the tail risks of unfavorable price movements for market participants in the Australian NEM, especially for those exposed to multiple states.

\footnotetext{
${ }^{16}$ The average absolute difference is calculated as $\frac{1}{M} \sum_{\ell=1}^{M}\left|p_{\ell}^{e}-p_{\ell}^{c}\right|$, where $M$ is the total number of considered joint probability, $p_{\ell}^{e}$ is the empirical joint probability, and $p_{\ell}^{c}$ is the simulated joint probability from either vine copula or multivariate Student's $t$ copula.
} 


\section{Discussion of the Results and Implications}

Based on the empirical results revealed by the R-vine copula method, we observed that the dependence structure and tail dependence in the NEM has evolved over the three periods, namely the pre-carbon period, the carbon tax period, and the post-carbon period. In this section, we briefly discussed the possible causes of such change and its implications.

During the pre-carbon period, the interconnectedness was relatively limited across the whole NEM. Roughly speaking, the NEM could be split into two major parts, a northern part represented by NSW/QLD and a southern part consisting of VIC/SA. ${ }^{17}$ Thus, the extreme price movements mainly stayed within either the northern or southern parts and were rarely synchronized across them. This evidence partially explains that we can observe the tail dependence in the VIC/SA and QLD/NSW pairs, but no tail dependence between VIC and QLD.

Over the carbon tax period, the dependence structure across different states remained the same, but the tail dependences (both UTD and LTD) decreased, especially for QLD/NSW and SA/VIC. There are potentially several factors contributing to such observation. Firstly, according to Nelson et al. (2010) in a survey of Australian carbon pass-through rates, state emission factors, including the contribution of wind generation, generated a variable set of outcomes, with VIC and NSW having the most significant emissions intensity factors of 1.23 and 0.90 , respectively. While the NSW black coal fleet is of older vintage, marginally more expensive, and has slightly higher carbon-emission intensity rates. Secondly, the policy of carbon tax has led to a higher average price, which further results in higher revenues for all generators.

Conversely, the cost of $\mathrm{CO}_{2}$ emission was partially offset by generous structural adjustment payments to the most affected coal generators, which can give them less incentive

\footnotetext{
${ }^{17} \mathrm{We}$ are grateful to the anonymous referee who pointed out such a split in the NEM during the pre-carbon period.
} 
to withhold capacity. Thirdly, as a direct consequence of carbon tax, there is a noticeable surge in supply from green energy, such as gas generation and hydro production. These changes in plant dispatch patterns could also contribute to changes in the tail dependence of NEM.

In the post-carbon period, the dependence structure had a significant change and reflected the geographical presence of the national electricity transmission lines. More importantly, there was a general increase in the tail dependences (both UTD and LTD), indicating that electricity prices in different states tended to have more extreme anomalies and occurred simultaneously. This resulted in a higher systemic risk associated with high contagion potential across electricity conditions in the Australian states. It is possible to explain this change by the pattern of generation. The carbon tax period witnessed the closure of a large number of coal plants (Simshauser, 2019). After the abolition of the carbon tax, the hydro output returned to the average level, and gas generators reduced their output and also needed to face sharply higher unit fuel costs after the commerce of LNG export. Such a change in the capacity and production of the plants seems to have reduced the amount of excess capacity in certain regions. Additionally, the loss of a few significant plants, especially during 2016 and 2017 , tightened the supply-demand mixture. The change in the pattern of generation reduced transmission congestion, and thus we can see the joint spikes more frequently.

Overall, the findings have substantial implications for risk management in the NEM electricity market. The presence of higher tail dependence, following the carbon tax regime, indicates a much higher risk for electricity market investments. Furthermore, the range of tail dependence is vital to risk-averse investors, who dislike risk and prefer to stay away from highrisk investments in their portfolios. Based on the fact that tail dependence can be treated as a tool for measuring the symmetric risk under extreme market situations, investors in the new era for NEM should consider tail dependence in asset pricing models, as well as in their investment decision process. The results stated in this paper can help understand the 
dependence structure across Australian state electricity markets, which is of great interest to policymakers and market participants. Moreover, the findings give a clear picture in relevance to the development of risk management and hedging strategies for electricity market participants, mainly for those operating in the considered markets across States. The findings could also be of substantial importance to policymakers planning to develop new guidelines for market mechanisms or to potential investors planning to construct new interconnectors.

\section{Conclusion}

This study used the R-vine copula methodological approach to investigate the dependence structure of electricity prices in five Australian states and aimed at shedding further light on the implication of risk management in the NEM. Through a structural break test, the analysis found strong evidence of breaks in the dependence of most state pairs due to the adoption and abolition of the carbon tax. More specifically, the analysis employed R-vine copula to investigate the dependence structure in three periods related to the adoption and abolition of the carbon tax. In the pre-carbon period, we found evidence of tail dependence separately in the northern and southern NEM, but not across them. During the carbon period, the joint spike in the northern NEM disappeared, and the tail dependence in the southern NEM decreased. In the post-carbon period, the best dependence structure turned out to be a flexible structure of the regular vine, which exactly matched the geographical infrastructure connectedness of transmission wires. Besides, both upper and lower tail dependences were found in all adjacent states after the abolition of the carbon tax, suggesting a more integrated market regarding tail dependence. From the market participants' perspective, this framework, based on the R-vine copula, can be used as a risk management tool, especially for identifying the tail risks of unfavorable price movements in multiple states. Future research on this subject can focus on 
the data at a high frequency to examine the dependence structure and system security across the electricity markets in the Australian states.

\section{References}

Aloui, R., Hammoudeh, S., \& Nguyen, D.K. (2013a). A time-varying copula approach to oil and stock market dependence: The case of transition economies. Energy Economics, 39, $208-221$.

Aloui, R., Aïssa, M.S.B., \& Nguyen, D.K. (2013b). Conditional dependence structure between oil prices and exchange rates: A copula-GARCH approach. Journal of International Money and Finance, 32, 719-738.

Apergis, N., Barunik, J., \& Lau, C.K.M. (2017a). Good volatility, bad volatility: What drives the asymmetric connectedness of Australian electricity markets? Energy Economics, 66, $108-116$.

Apergis, N., Fontini, F., \&Inchauspe, J. (2017b). Integration of regional electricity markets in Australia: A price convergence assessment. Energy Economics, 62, 411-418.

Apergis, N., Gozgor, G., Lau, C.K.M., \& Wang, S. (2019). Decoding the Australian electricity market: New evidence from three-regime hidden semi-Marko model. Energy Economics, 78, 129-142.

Bedford, T., \& Cooke, R.M. (2001). Probability density decomposition for conditionally dependent random variables modeled by vines. Annals of Mathematics and Artificial Intelligence, 32(1-4), 245-268.

Bedford, T., \& Cooke, R.M. (2002). Vines-a new graphical model for dependent random variables. The Annals of Statistics, 30(4), 1031-1068. 
Bell, W.P., Wild, P., Foster, J., \& Hewson, M. (2017). Revitalising the wind power induced merit order effect to reduce wholesale and retail electricity prices in Australia. Energy Economics, 67, 224-241.

Brechmann, E.C., Czado, C., \&Aas, K. (2012). Truncated regular vines in high dimensions with application to financial data. Canadian Journal of Statistics, 40(1), 68-85.

Cutler, N.J., Boerema, N.D., MacGill, I.F., \&Outhred, H.R. (2011). High penetration wind generation impacts on spot prices in the Australian national electricity market. Energy Policy, 39(10), 5939-5949.

Czado, C. (2010). Pair-copula constructions of multivariate copulas. In: Copula Theory and its Applications, (pp. 93-109), Springer Berlin: Heidelberg.

Czado, C., Gärtner, F., \& Min, A. (2010). Analysis of Australian electricity loads using joint Bayesian inference of D-Vines with autoregressive margins. In: Dependence Modeling: Vine Copula Handbook, (pp. 265-280), World Scientific: Singapore.

Creal, D., Koopman, S.J., \& Lucas, A. (2013). Generalized autoregressive score models with applications. Journal of Applied Econometrics, 28(5), 777-795.

Dißmann, J., Brechmann, E.C., Czado, C., \&Kurowicka, D. (2013). Selecting and estimating regular vine copulae and application to financial returns. Computational Statistics \& Data Analysis, 59, 52-69.

Fisher, T.J., \& Gallagher, C.M. (2012). New weighted portmanteau statistics for time series goodness of fit testing. Journal of the American Statistical Association, 107(498), 777787.

Genest, C., \& Favre, A.C. (2007). Everything you always wanted to know about copula modeling but were afraid to ask. Journal of Hydrologic Engineering, 12(4), 347-368.

Hansen, B.E. (1994). Autoregressive conditional density estimation. International Economic Review, 35(3), 705-730. 
Higgs, H. (2009). Modelling price and volatility inter-relationships in the Australian wholesale spot electricity markets. Energy Economics, 31(5), 748-756.

Higgs, H., \& Worthington, A. (2008). Stochastic price modeling of high volatility, meanreverting, spike-prone commodities: The Australian wholesale spot electricity market. Energy Economics, 30(6), 3172-3185.

Hu, X., Grozev, G., \& Batten, D. (2005). Empirical observations of bidding patterns in Australia's National Electricity Market. Energy Policy, 33(16), 2075-2086.

Huisman, R., \& Mahieu, R. (2003). Regime jumps in electricity prices. Energy Economics, 25(5), 425-434.

Joe, H. (1996). Families of $\mathrm{m}$-variate distributions with given margins and $\mathrm{m}(\mathrm{m}-1) / 2$ bivariate dependence parameters Lecture Notes-Monograph Series, 28, 120-141.

Joe, H., \&Kurowicka, D. (Eds.). (2011). Dependence Modeling: Vine Copula Handbook. World Scientific: Singapore.

Kurowicka, D., \& Cooke, R.M. (2006). Uncertainty Analysis with High Dimensional Dependence Modelling. John Wiley \& Sons: New York.

Li, X., \& Wei, Y. (2018). The dependence and risk spillover between crude oil market and China stock market: New evidence from a variational mode decomposition-based copula method. Energy Economics, 74, 565-581.

Lucia, J.J., \& Schwartz, E.S. (2002). Electricity prices and power derivatives: Evidence from the Nordic power exchange. Review of Derivatives Research, 5(1), 5-50.

Manner, H., Fard, F.A., Pourkhanali, A., \&Tafakori, L. (2019). Forecasting the joint distribution of Australian electricity prices using dynamic vine copulae. Energy Economics, 78, 143-164.

Nagler, T., Schepsmeier, U., Stoeber, J., Brechmann, E.C., Graeler, B., Erhardt, T., Almeida, C., Min, A., Czado, C., Hofmann, M., Killiches, M., Joe, H., and Vatter, T. (2019). 
VineCopula: Statistical Inference of Vine Copulas. $\mathrm{R}$ package version 2.2.0. https://CRAN.R-project.org/package=VineCopula.

Nelsen, R.B. (1999). An Introduction to Copulas. Springer-Verlag: New York.

Nelson, T., Orton, F., \&Kelley, S. (2010). The impact of carbon pricing on Australian deregulated wholesale electricity and gas markets.AGL Applied Economic \& Policy Research Working Paper, No. 23.

Ning, C. (2010). Dependence structure between the equity market and the foreign exchange market-a copula approach. Journal of International Money and Finance, 29(5), 743759.

Pircalabu, A., Hvolby, T., Jung, J., \&Høg, E. (2017). Joint price and volumetric risk in wind power trading: A copula approach. Energy Economics, 62, 139-154.

Patton, A.J. (2012). A review of copula models for economic time series. Journal of Multivariate Analysis, 110, 4-18.

Patton, A.J. (2013). Copula methods for forecasting multivariate time series. In: Handbook of Economic Forecasting, Vol. 2, (pp. 899-960), Elsevier: Amsterdam.

Reboredo, J.C. (2011). How do crude oil prices co-move? A copula approach. Energy Economics, 33(5), 948-955.

Reboredo, J.C. (2015). Is there dependence and systemic risk between oil and renewable energy stock prices? Energy Economics, 48, 32-45.

Reboredo, J.C., Rivera-Castro, M.A., \& Zebende, G.F. (2014). Oil and US dollar exchange rate dependence: A detrended cross-correlation approach. Energy Economics, 42, 132-139.

Simshauser, P. (2018). On intermittent renewable generation and the stability of Australia's National Electricity Market. Energy Economics, 72, 1-19. 
Simshauser, P. (2019). Lessons from Australia's National Electricity Market 1998-2018: The strengths and weaknesses of the reform experience. University of Cambridge Working Paper, No. 1972.

Simshauser, P., Tian, Y., \& Whish-Wilson, P. (2015). Vertical integration in energy-only electricity markets. Economic Analysis and Policy, 48, 35-56.

Sukcharoen, K., \&Leatham, D.J. (2017). Hedging downside risk of oil refineries: A vine copula approach. Energy Economics, 66, 493-507.

Stöeber, J., Joe, H., \&Czado, C. (2013). Simplified pair copula constructions-limitations and extensions. Journal of Multivariate Analysis, 119, 101-118.

Wang, Y.C., Wu, J.L., \& Lai, Y.H. (2013). A revisit to the dependence structure between the stock and foreign exchange markets: A dependence-switching copula approach. Journal of Banking and Finance, 37(5), 1706-1719.

Wen, X., Wei, Y., \& Huang, D. (2012). Measuring contagion between energy market and stock market during financial crisis: A copula approach. Energy Economics, 34(5), 14351446.

Wood, T., \& Blowers, D. (2017). Next generation: the long-term future of the National Electricity Market. Grattan Institute Working Paper, September 2017. 
Appendix. A.

Table A1. Estimation of Deterministic Component in Electricity Price.

\begin{tabular}{|c|c|c|c|c|c|c|c|c|c|c|}
\hline & \multicolumn{2}{|c|}{ QLD } & \multicolumn{2}{|c|}{ NSW } & \multicolumn{2}{|c|}{ VIC } & \multicolumn{2}{|c|}{ SA } & \multicolumn{2}{|c|}{ TAS } \\
\hline & Para Est. & $p$-value & Para Est. & $p$-value & Para Est. & $p$-value & Para Est. & $p$-value & Para Est. & $p$-value \\
\hline (Intercept) & 64.20 & $0.00 \%$ & 43.30 & $0.00 \%$ & 70.89 & $0.00 \%$ & 97.74 & $0.00 \%$ & 63.41 & $0.00 \%$ \\
\hline day_Tuesday & -0.60 & $87.73 \%$ & 1.06 & $74.90 \%$ & -0.27 & $95.37 \%$ & -1.60 & $80.64 \%$ & 0.80 & $73.43 \%$ \\
\hline day_Wednesday & 3.38 & $38.80 \%$ & 0.24 & $94.19 \%$ & 1.73 & $70.49 \%$ & -0.17 & $97.93 \%$ & 0.10 & $96.53 \%$ \\
\hline day_Thursday & 6.48 & $9.80 \%$ & 2.30 & $48.65 \%$ & 13.38 & $0.34 \%$ & 11.11 & $8.85 \%$ & 0.44 & $85.27 \%$ \\
\hline day_Friday & 1.05 & $78.89 \%$ & 6.04 & $6.79 \%$ & 0.61 & $89.35 \%$ & -4.58 & $48.25 \%$ & -0.12 & $95.79 \%$ \\
\hline day_Saturday & -6.02 & $12.47 \%$ & -7.52 & $2.30 \%$ & -9.20 & $4.40 \%$ & -20.05 & $0.21 \%$ & -4.45 & $5.94 \%$ \\
\hline day_Sunday & -9.60 & $1.43 \%$ & -10.11 & $0.22 \%$ & -12.06 & $0.83 \%$ & -23.22 & $0.04 \%$ & -4.67 & $4.79 \%$ \\
\hline month_Feb & -3.13 & $54.72 \%$ & 12.94 & $0.33 \%$ & -24.51 & $0.01 \%$ & -31.35 & $0.03 \%$ & -0.10 & $97.38 \%$ \\
\hline month_Mar & -26.02 & $0.00 \%$ & -9.61 & $2.52 \%$ & -30.24 & $0.00 \%$ & -49.35 & $0.00 \%$ & 7.81 & $1.07 \%$ \\
\hline month_Apr & -36.13 & $0.00 \%$ & -4.25 & $32.58 \%$ & -25.60 & $0.00 \%$ & -47.66 & $0.00 \%$ & 10.90 & $0.04 \%$ \\
\hline month_May & -36.40 & $0.00 \%$ & -5.57 & $19.41 \%$ & -27.99 & $0.00 \%$ & -43.99 & $0.00 \%$ & -2.74 & $37.48 \%$ \\
\hline month_Jun & -31.44 & $0.00 \%$ & 1.45 & $73.74 \%$ & -21.78 & $0.03 \%$ & -35.63 & $0.00 \%$ & 9.36 & $0.26 \%$ \\
\hline month_Jul & -37.51 & $0.00 \%$ & -5.97 & $16.45 \%$ & -28.57 & $0.00 \%$ & -28.71 & $0.07 \%$ & -10.02 & $0.12 \%$ \\
\hline month_Aug & -39.32 & $0.00 \%$ & -6.79 & $11.34 \%$ & -33.82 & $0.00 \%$ & -51.47 & $0.00 \%$ & -12.90 & $0.00 \%$ \\
\hline month_Sep & -39.57 & $0.00 \%$ & -8.12 & $6.07 \%$ & -35.62 & $0.00 \%$ & -55.65 & $0.00 \%$ & -16.25 & $0.00 \%$ \\
\hline month_Oct & -39.79 & $0.00 \%$ & -6.31 & $14.17 \%$ & -38.09 & $0.00 \%$ & -58.02 & $0.00 \%$ & -12.86 & $0.00 \%$ \\
\hline month_Nov & -31.35 & $0.00 \%$ & 0.19 & $96.56 \%$ & -33.46 & $0.00 \%$ & -40.09 & $0.00 \%$ & -5.14 & $9.86 \%$ \\
\hline month_Dec & -30.70 & $0.00 \%$ & -6.08 & $15.68 \%$ & -39.03 & $0.00 \%$ & -52.84 & $0.00 \%$ & -12.93 & $0.00 \%$ \\
\hline year_2009 & -0.71 & $88.45 \%$ & 5.34 & $19.80 \%$ & -5.54 & $33.34 \%$ & 4.33 & $59.62 \%$ & -29.10 & $0.00 \%$ \\
\hline year_2010 & -3.05 & $53.41 \%$ & -2.11 & $61.02 \%$ & -14.76 & $0.99 \%$ & -18.44 & $2.42 \%$ & -29.03 & $0.00 \%$ \\
\hline year_2011 & -4.99 & $30.92 \%$ & -9.22 & $2.61 \%$ & -14.52 & $1.11 \%$ & -20.68 & $1.14 \%$ & -25.89 & $0.00 \%$ \\
\hline year_2012 & 33.04 & $0.00 \%$ & 16.28 & $0.01 \%$ & 15.66 & $0.63 \%$ & 18.83 & $2.13 \%$ & -10.17 & $0.06 \%$ \\
\hline year_2013 & 24.42 & $0.00 \%$ & 13.40 & $0.12 \%$ & 9.67 & $9.11 \%$ & 10.73 & $18.92 \%$ & -16.50 & $0.00 \%$ \\
\hline year_2014 & 18.52 & $0.02 \%$ & -3.68 & $37.47 \%$ & -11.47 & $4.52 \%$ & -11.69 & $15.29 \%$ & -21.32 & $0.00 \%$ \\
\hline year_2015 & 25.88 & $0.00 \%$ & 12.70 & $0.22 \%$ & 4.27 & $45.55 \%$ & 10.62 & $19.38 \%$ & 7.41 & $2.65 \%$ \\
\hline
\end{tabular}




\begin{tabular}{ccccccccccc} 
year_2016 & 59.12 & $\mathbf{0 . 0 0 \%}$ & 42.36 & $\mathbf{0 . 0 0 \%}$ & 24.76 & $\mathbf{0 . 0 0 \%}$ & 57.69 & $\mathbf{0 . 0 0 \%}$ & 16.93 & $\mathbf{0 . 0 0 \%}$ \\
year_2017 & 38.88 & $\mathbf{0 . 0 0 \%}$ & 43.44 & $\mathbf{0 . 0 0 \%}$ & 50.54 & $\mathbf{0 . 0 0 \%}$ & 47.17 & $\mathbf{0 . 0 0 \%}$ & 28.51 & $\mathbf{0 . 0 0 \%}$ \\
year_2018 & 46.32 & $\mathbf{0 . 0 0 \%}$ & 49.74 & $\mathbf{0 . 0 0 \%}$ & 68.03 & $\mathbf{0 . 0 0 \%}$ & 58.88 & $\mathbf{0 . 0 0 \%}$ & 31.55 & $\mathbf{0 . 0 0 \%}$ \\
\hline outage & & & & & & & & 4017 & & \\
\hline No of Obs. & 4017 & & 4017 & & 4017 & & 708.65 & $\mathbf{0 . 0 0 \%}$ \\
Adj. R2 & $11.93 \%$ & & $12.09 \%$ & & $11.54 \%$ & & $7.81 \%$ & $38.96 \%$ & \\
F-stat & 21.15 & & 21.46 & & 20.41 & & 13.60 & 92.55 \\
\hline
\end{tabular}


QLD
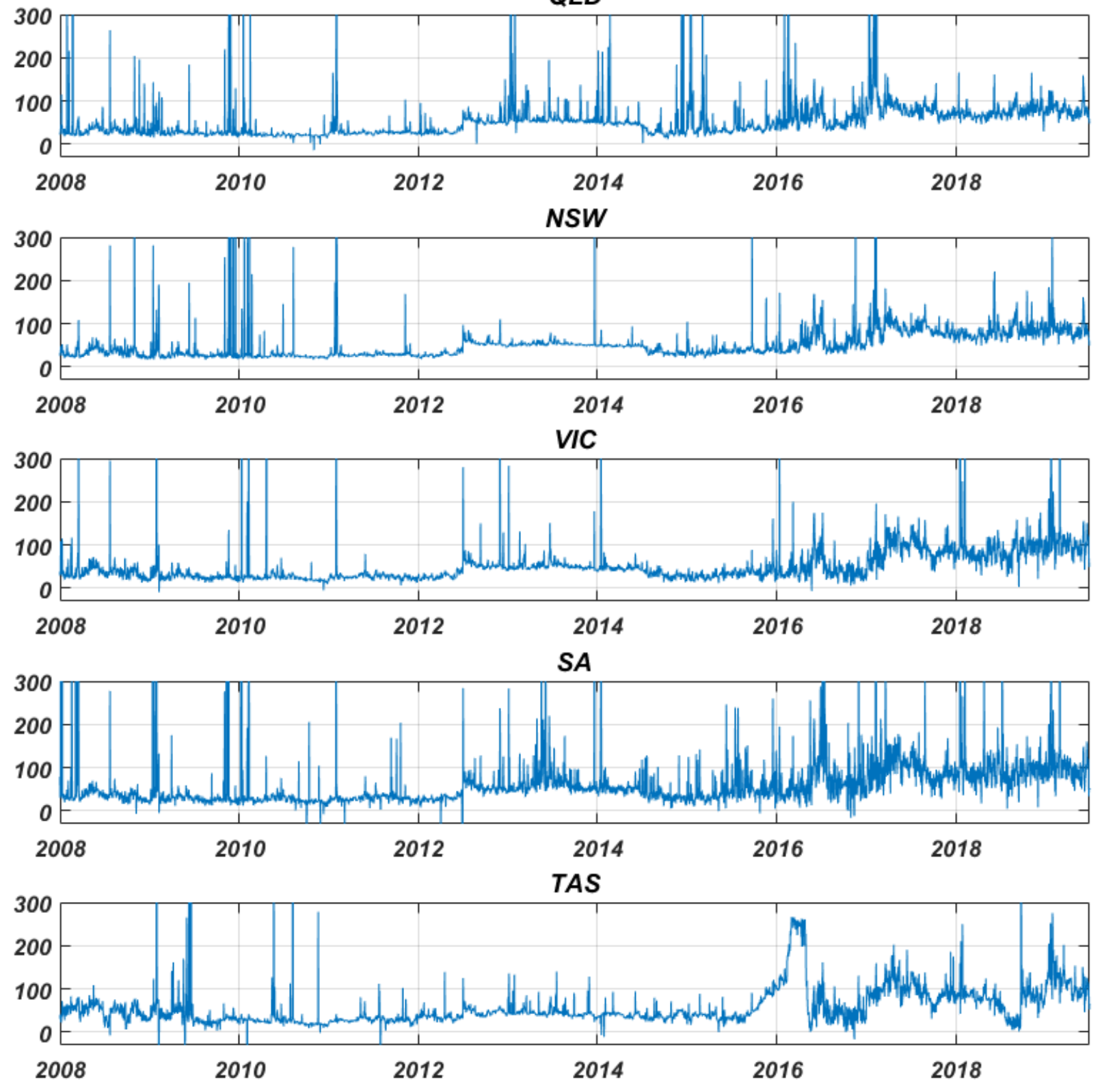

Figure 1. Electricity Price in NEM (the Y-axis is Limited between -50 and 300). 
$\begin{array}{llllll}0.0 & 0.2 & 0.4 & 0.6 & 0.8 & 1.0\end{array}$
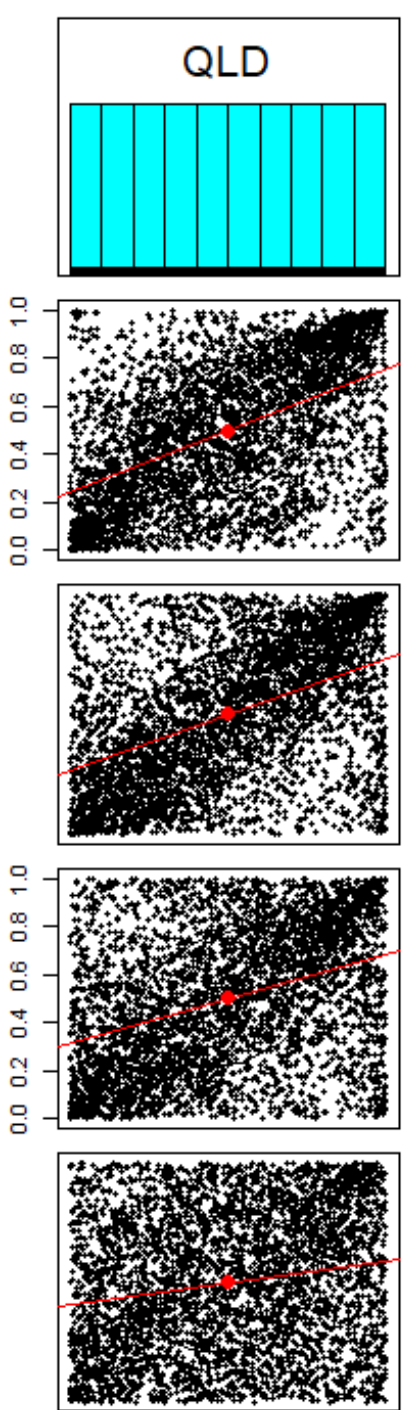

$\begin{array}{llllll}0.0 & 0.2 & 0.4 & 0.6 & 0.8 & 1.0\end{array}$
0.38

NSW
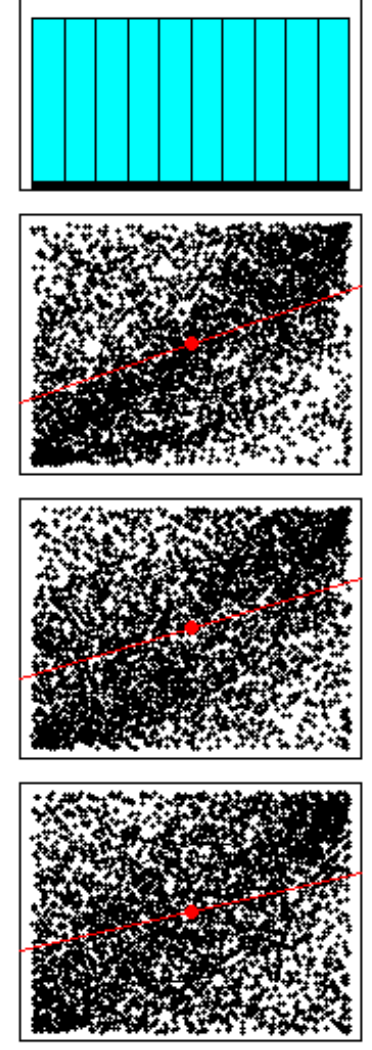

$\begin{array}{llllll}0.0 & 0.2 & 0.4 & 0.6 & 0.8 & 1.0\end{array}$

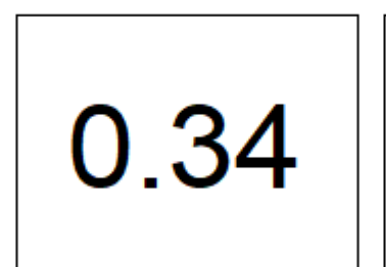

0.32

0.27

0.21

0.55

SA
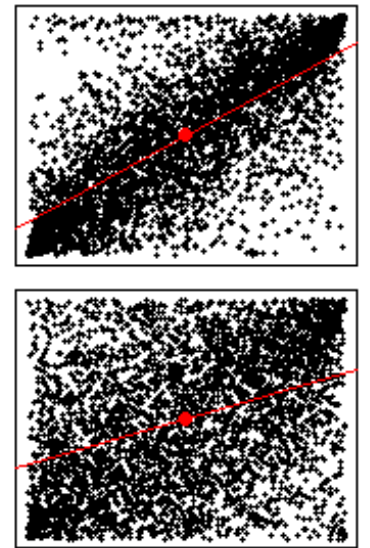

$\begin{array}{llllll}0.0 & 0.2 & 0.4 & 0.6 & 0.8 & 1.0\end{array}$

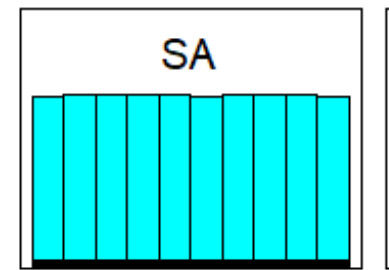

0.21
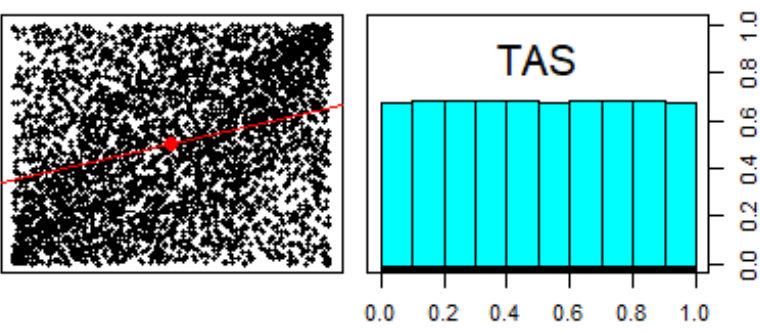

Figure 2. Pairwise Plots of PIT Variables and their Kendall's tau. 

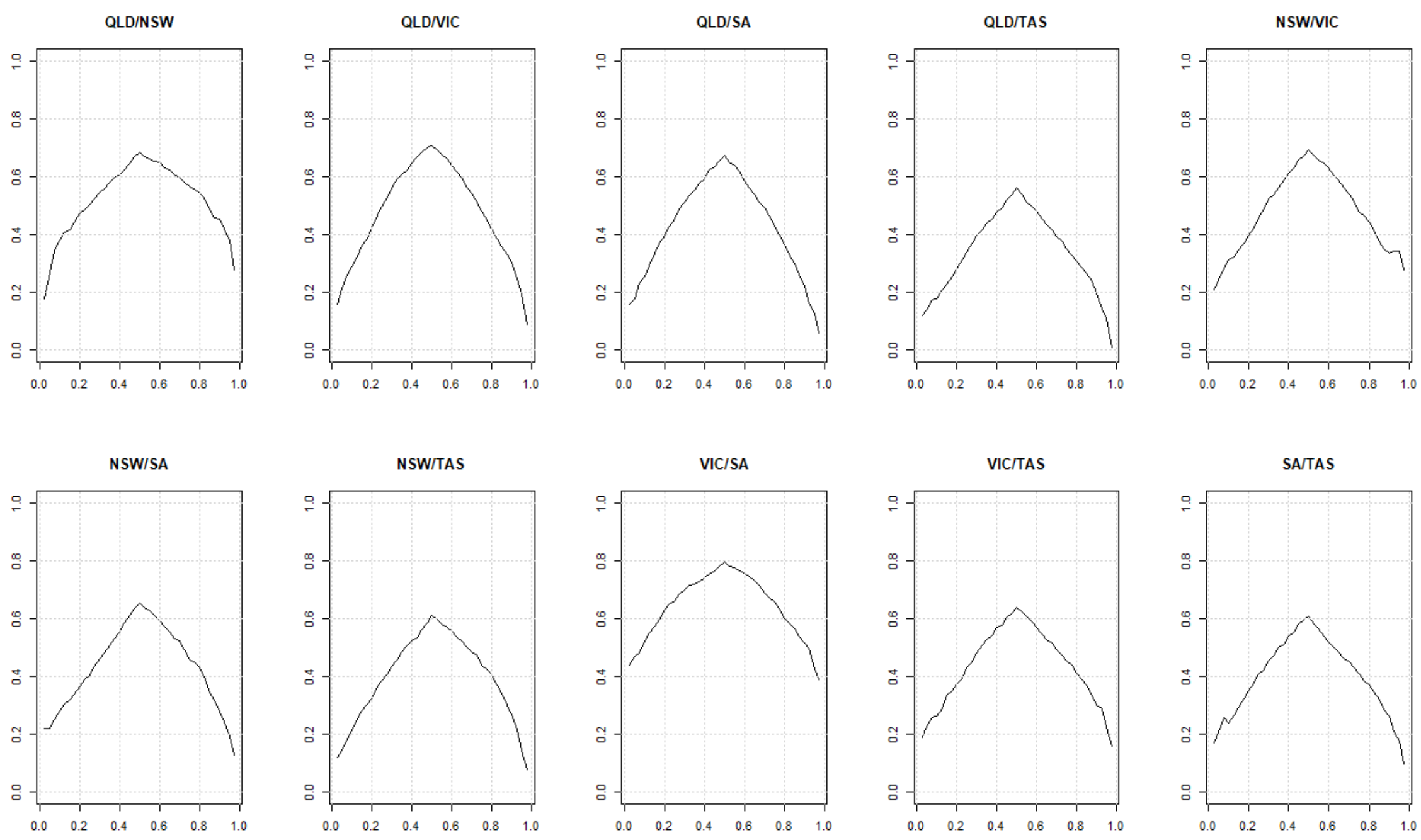

Figure 3. Quantile Dependence for All Pairs. 
Period 1: July 2008 - June 2012

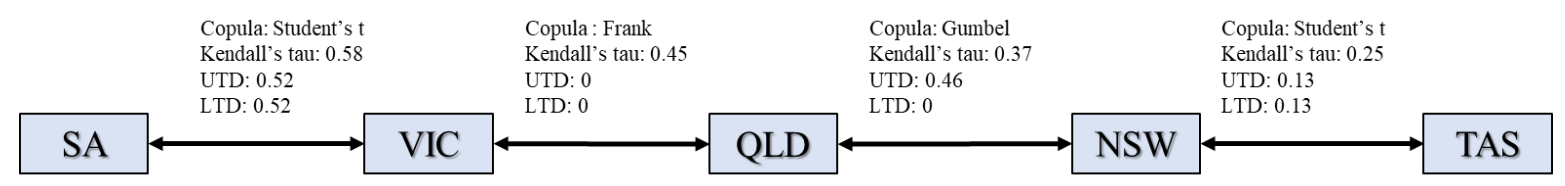

Period 2: July 2012 - June 2014

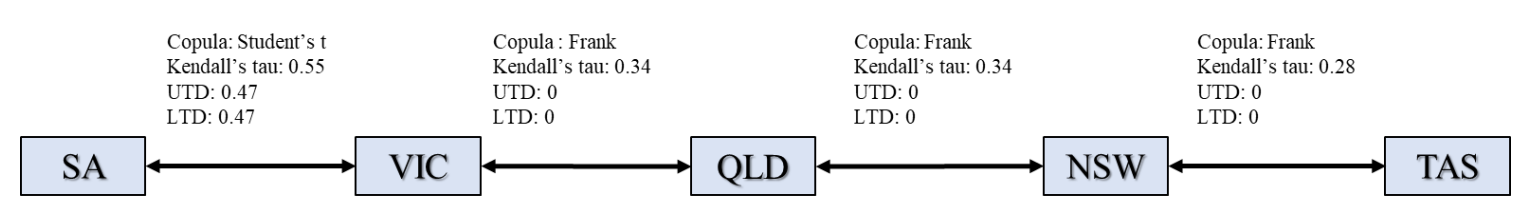

Period 3: July 2014 - June 2019

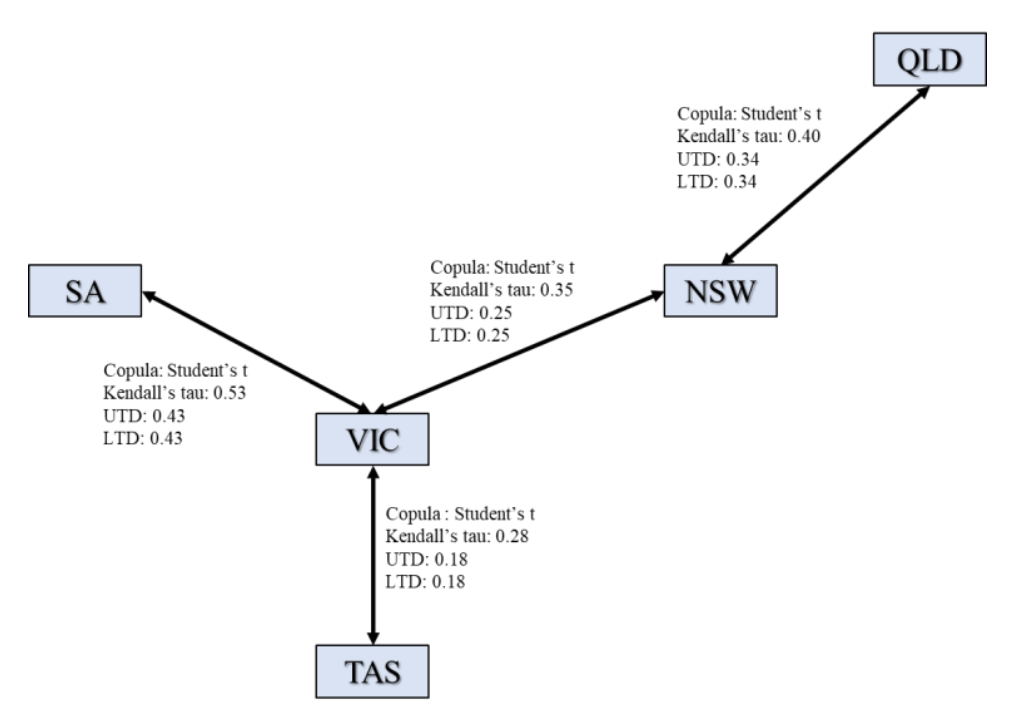

Figure 4. Tree 1 of R-vine Copula in Three Periods. 


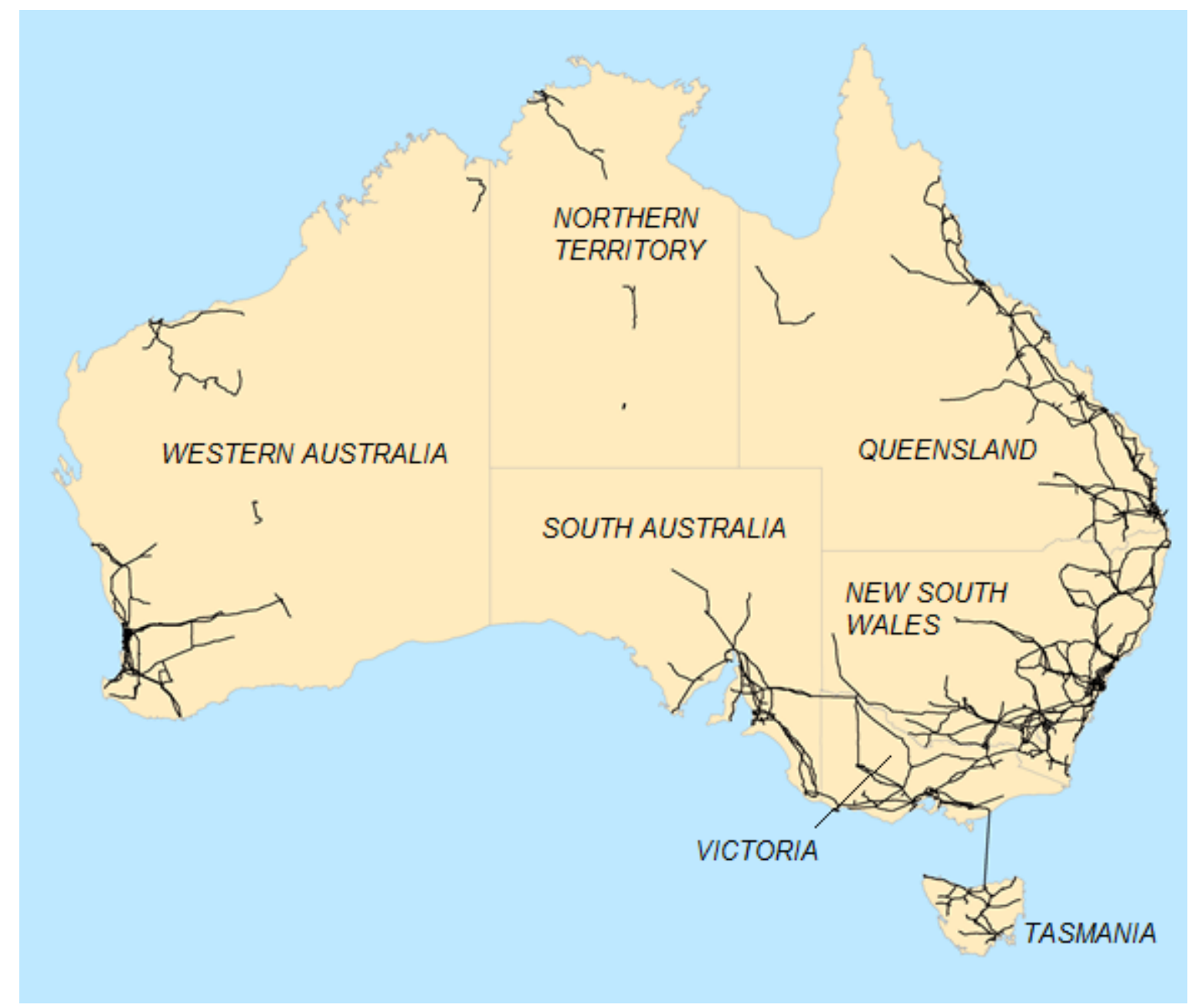

Source: Geoscience Australia, 2015

Figure 5. Australian National Electricity Transmission Lines 
Table 1

Descriptive Statistics

\begin{tabular}{cccccc}
\hline \multicolumn{7}{c}{ QLD } & NSW & VIC & SA & TAS \\
\hline Moments & & & \\
Mean & 55.44 & 53.36 & 52.93 & 66.71 & 57.45 \\
Skewness & 75.77 & 58.60 & 80.77 & 127.18 & 50.07 \\
Kurtosis & 13.20 & 13.87 & 25.03 & 12.92 & 5.06 \\
Min & 239.28 & 265.72 & 879.37 & 226.46 & 53.79 \\
\hline \multicolumn{7}{c}{ Distribution } \\
10\% Quantile & -13.98 & 16.80 & -8.94 & -103.16 & -181.69 \\
30\% Quantile & 22.18 & 24.33 & 21.79 & 22.99 & 23.42 \\
Median & 28.74 & 30.71 & 28.87 & 31.71 & 33.16 \\
70\% Quantile & 45.03 & 44.12 & 40.55 & 45.20 & 42.44 \\
90\% Quantile & 59.96 & 55.71 & 53.32 & 64.52 & 61.53 \\
Max & 88.25 & 88.95 & 96.84 & 109.51 & 105.20 \\
\hline \multicolumn{7}{c}{ Frequency of Price Anomalies } \\
Fq. of Positive Spikes & $1.41 \%$ & $1.02 \%$ & $0.74 \%$ & $1.45 \%$ & $1.33 \%$ \\
Fq. of Negative Prices & $0.02 \%$ & $0.00 \%$ & $0.07 \%$ & $0.38 \%$ & $0.33 \%$ \\
\hline
\end{tabular}

Table 2

Correlation and Dependence

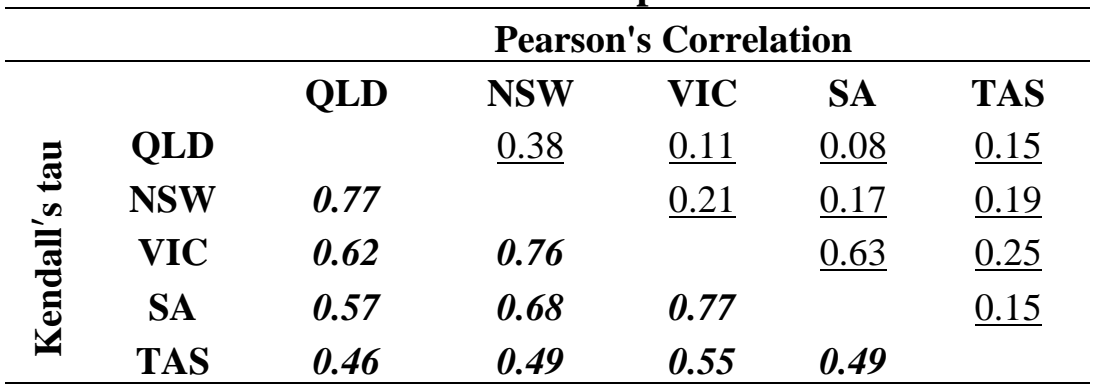

Note: Pearson correlation is shown with underscore above the main diagonal.

Kendall's tau is shown in bold and Italic font below the main diagonal. 
Table 3

Parameter Estimation in Marginal Model

\begin{tabular}{|c|c|c|c|c|c|c|c|c|c|c|}
\hline & \multicolumn{2}{|c|}{ QLD } & \multicolumn{2}{|c|}{ NSW } & \multicolumn{2}{|c|}{ VIC } & \multicolumn{2}{|c|}{ SA } & \multicolumn{2}{|c|}{ TAS } \\
\hline & Para Est. & $p$-value & Para Est. & $p$-value & Para Est. & $p$-value & Para Est. & $p$-value & Para Est. & $p$-value \\
\hline \multicolumn{11}{|c|}{ Conditional mean } \\
\hline $\operatorname{ar1}$ & 1.44 & $0.00 \%$ & 0.36 & $0.00 \%$ & -0.12 & $6.17 \%$ & 1.17 & $0.00 \%$ & 0.99 & $0.00 \%$ \\
\hline $\operatorname{ar2}$ & -0.45 & $0.00 \%$ & -0.26 & $0.00 \%$ & -0.13 & $0.01 \%$ & -0.69 & $0.00 \%$ & & \\
\hline $\operatorname{ar} 3$ & & & 0.39 & $0.00 \%$ & 0.53 & $0.00 \%$ & 0.30 & $0.00 \%$ & & \\
\hline ar4 & & & 0.04 & $1.83 \%$ & 0.53 & $0.00 \%$ & -0.03 & $22.15 \%$ & & \\
\hline $\operatorname{ar5}$ & & & 0.02 & $13.88 \%$ & 0.09 & $1.29 \%$ & -0.02 & $32.84 \%$ & & \\
\hline ar6 & & & 0.06 & $0.00 \%$ & & & 0.16 & $0.00 \%$ & & \\
\hline ar7 & & & 0.24 & $0.00 \%$ & & & & & & \\
\hline ma1 & -0.79 & $0.00 \%$ & 0.31 & $0.00 \%$ & 0.78 & $0.00 \%$ & -0.63 & $0.00 \%$ & -0.34 & $0.00 \%$ \\
\hline ma2 & & & 0.36 & $0.00 \%$ & 0.57 & $0.00 \%$ & 0.30 & $0.00 \%$ & -0.18 & $0.00 \%$ \\
\hline ma3 & & & & & -0.12 & $0.00 \%$ & & & -0.04 & $0.14 \%$ \\
\hline ma4 & & & & & -0.54 & $0.00 \%$ & & & & \\
\hline ma5 & & & & & -0.38 & $0.00 \%$ & & & & \\
\hline ma6 & & & & & -0.21 & $0.00 \%$ & & & & \\
\hline \multicolumn{11}{|c|}{$\underline{\text { Conditional variance }}$} \\
\hline omega & 51.89 & $0.00 \%$ & 15.35 & $0.00 \%$ & 36.59 & $0.00 \%$ & 180.24 & $0.00 \%$ & 14.74 & $0.00 \%$ \\
\hline alpha & 0.70 & $0.00 \%$ & 0.45 & $0.00 \%$ & 0.38 & $0.00 \%$ & 0.48 & $0.00 \%$ & 0.50 & $0.00 \%$ \\
\hline aeta & 0.30 & $0.00 \%$ & 0.55 & $0.00 \%$ & 0.60 & $0.00 \%$ & 0.52 & $0.00 \%$ & 0.49 & $0.00 \%$ \\
\hline \multicolumn{11}{|c|}{$\underline{\text { Error distribution - skewedt }}$} \\
\hline shape & 2.90 & $0.00 \%$ & 3.04 & $0.00 \%$ & 2.90 & $0.00 \%$ & 2.67 & $0.00 \%$ & 2.98 & $0.00 \%$ \\
\hline skew & 1.34 & $0.00 \%$ & 1.34 & $0.00 \%$ & 1.05 & $0.00 \%$ & 1.19 & $0.00 \%$ & 1.15 & $0.00 \%$ \\
\hline
\end{tabular}


Table 4

P-values of Three Diagnostic Tests on Standardized Residuals from Marginal Models

Weighted Ljung-Box Test on Standardized Residuals

\begin{tabular}{cccc} 
Lag Length & Lag-1 & Lag-2 & Lag-3 \\
\hline QLD & $92.37 \%$ & $18.83 \%$ & $38.76 \%$ \\
NSW & $71.34 \%$ & $6.32 \%$ & $16.62 \%$ \\
VIC & $89.51 \%$ & $8.58 \%$ & $19.26 \%$ \\
SA & $63.49 \%$ & $6.43 \%$ & $8.78 \%$ \\
TAS & $32.41 \%$ & $11.66 \%$ & $8.71 \%$ \\
\hline
\end{tabular}

Weighted Ljung-Box Test on Standardized Squared Residuals

\begin{tabular}{cccc} 
Lag Length & Lag-1 & Lag-2 & Lag-3 \\
\hline QLD & $99.94 \%$ & $99.87 \%$ & $100.00 \%$ \\
NSW & $99.97 \%$ & $99.93 \%$ & $100.00 \%$ \\
VIC & $99.89 \%$ & $99.69 \%$ & $99.99 \%$ \\
SA & $99.86 \%$ & $99.65 \%$ & $99.99 \%$ \\
TAS & $99.68 \%$ & $99.19 \%$ & $99.97 \%$ \\
\hline
\end{tabular}

Weighted ARCH LM Tests

\begin{tabular}{cccc} 
Lag Length & $\mathbf{3}$ & $\mathbf{4}$ & $\mathbf{7}$ \\
\hline QLD & $99.94 \%$ & $100.00 \%$ & $100.00 \%$ \\
NSW & $99.97 \%$ & $100.00 \%$ & $100.00 \%$ \\
VIC & $99.89 \%$ & $100.00 \%$ & $100.00 \%$ \\
SA & $99.86 \%$ & $100.00 \%$ & $100.00 \%$ \\
TAS & $99.68 \%$ & $99.98 \%$ & $100.00 \%$ \\
\hline
\end{tabular}

Note: Lag-1 is always 1, Lag-2 is $3 \times(p+q)-1$, and Lag-3 is $5 \times(p+q)-1$, where $p$ and $q$ are the orders in the marginal models of $\operatorname{ARMA}(p, q)-\operatorname{GARCH}(1,1)$. 


\section{Table 5}

Structural Break Test for Pairwise Dependence with Known Dates

\begin{tabular}{lcc}
\hline \multicolumn{1}{c}{ Break Date: } & July 1, 2012 & July 1, 2014 \\
\hline QLD/NSW & $65.50 \%$ & $\mathbf{4 . 1 0 \%}$ \\
QLD/VIC & $\mathbf{0 . 0 0 \%}$ & $\mathbf{0 . 0 0 \%}$ \\
QLD/SA & $\mathbf{0 . 0 0 \%}$ & $\mathbf{0 . 0 0 \%}$ \\
QLD/TAS & $\mathbf{0 . 3 0 \%}$ & $\mathbf{0 . 1 0 \%}$ \\
NSW/VIC & $\mathbf{0 . 6 0 \%}$ & $\mathbf{0 . 3 0 \%}$ \\
NSW/SA & $60.10 \%$ & $84.40 \%$ \\
NSW/TAS & $\mathbf{0 . 2 0 \%}$ & $\mathbf{0 . 0 0 \%}$ \\
VIC/SA & $\mathbf{0 . 7 0 \%}$ & $\mathbf{1 . 3 0 \%}$ \\
VIC/TAS & $22.50 \%$ & $11.40 \%$ \\
SA/TAS & $92.50 \%$ & $39.10 \%$ \\
\hline
\end{tabular}


Table 6

Estimates of the Vine Copula Model

\begin{tabular}{|c|c|c|c|c|c|c|c|c|c|}
\hline \multicolumn{10}{|c|}{ Period 1: July 2008 - June 2012} \\
\hline Tree & Edge & Copula & Para1 & std. error & Para2 & std. error & Kendall's tau & LTD & UTD \\
\hline \multirow{4}{*}{1} & NSW,TAS & Student's t & 0.38 & $(0.02)$ & 5.5 & $(0.92)$ & 0.25 & 0.13 & 0.13 \\
\hline & QLD,NSW & Gumbel & 1.6 & $(0.03)$ & & & 0.37 & 0.46 & \\
\hline & VIC,QLD & Frank & 4.95 & $(0.20)$ & & & 0.45 & & \\
\hline & SA,VIC & Student's t & 0.79 & $(0.01)$ & 3.21 & $(0.31)$ & 0.58 & 0.52 & 0.52 \\
\hline \multirow{3}{*}{2} & QLD,TAS|NSW & Survival Joe & 1.07 & $(0.02)$ & & & 0.04 & & 0.09 \\
\hline & VIC,NSW|QLD & Student's t & 0.1 & $(0.03)$ & 6.34 & (1.16) & 0.07 & 0.04 & 0.04 \\
\hline & SA,QLD|VIC & Frank & 1.54 & $(0.17)$ & & & 0.17 & & \\
\hline \multirow{2}{*}{3} & VIC,TAS|QLD,NSW & Frank & 1.86 & $(0.17)$ & & & 0.2 & & \\
\hline & SA,NSW|VIC,QLD & Frank & 0.76 & $(0.16)$ & & & 0.08 & & \\
\hline 4 & SA,TAS|VIC,QLD,NSW & \multicolumn{2}{|l|}{ Independent } & & & & 0.00 & & \\
\hline & Type: D-vine & \multicolumn{2}{|c|}{ LogLik: 1612.15} & \multicolumn{2}{|c|}{ AIC: -3200.31} & \multicolumn{2}{|c|}{ BIC: -3136.86} & & \\
\hline \multicolumn{10}{|c|}{ Period 2: July 2012 - June 2014} \\
\hline Tree & Edge & Copula & Para1 & std. error & Para2 & std. error & Kendall's tau & LTD & UTD \\
\hline \multirow{4}{*}{1} & NSW,TAS & Frank & 2.66 & $(0.24)$ & & & 0.28 & & \\
\hline & QLD,NSW & Frank & 3.33 & $(0.25)$ & & & 0.34 & & \\
\hline & VIC,QLD & Frank & 3.39 & $(0.26)$ & & & 0.34 & & \\
\hline & SA,VIC & Student's t & 0.76 & $(0.02)$ & 3.53 & $(0.55)$ & 0.55 & 0.47 & 0.47 \\
\hline \multirow{3}{*}{2} & QLD,TAS|NSW & Independent & & & & & 0.00 & & \\
\hline & VIC,NSW|QLD & Student's t & 0.26 & $(0.04)$ & 5.52 & $(1.27)$ & 0.17 & 0.10 & 0.10 \\
\hline & SA,QLD|VIC & Independent & & & & & & & \\
\hline \multirow[t]{2}{*}{3} & VIC,TAS|QLD,NSW & Student's t & 0.24 & $(0.04)$ & 4.84 & $(0.90)$ & 0.15 & 0.11 & 0.11 \\
\hline & SA,NSW|VIC,QLD & Frank & 1.36 & $(0.23)$ & & & 0.15 & & \\
\hline 4 & SA,TAS|VIC,QLD,NSW & \multicolumn{4}{|l|}{ Independent } & \multicolumn{4}{|c|}{0.00} \\
\hline & Type: D-vine & \multicolumn{2}{|c|}{ LogLik: 653.82} & \multicolumn{2}{|c|}{ AIC: -1287.63} & \multicolumn{2}{|c|}{ BIC: -1241.7} & & \\
\hline \multicolumn{10}{|c|}{ Period 3: July 2014 - June 2019} \\
\hline Tree & Edge & Copula & Para1 & std. error & Para2 & std. error & Kendall's tau & LTD & UTD \\
\hline \multirow{4}{*}{1} & NSW,QLD & Student's t & 0.59 & $(0.02)$ & 3.32 & $(0.33)$ & 0.4 & 0.34 & 0.34 \\
\hline & VIC,NSW & Student's t & 0.53 & $(0.02)$ & 4.46 & $(0.57)$ & 0.35 & 0.25 & 0.25 \\
\hline & VIC,SA & Student's t & 0.74 & $(0.01)$ & 3.79 & $(0.42)$ & 0.53 & 0.43 & 0.43 \\
\hline & TAS,VIC & Student's t & 0.42 & $(0.02)$ & 4.73 & $(0.62)$ & 0.28 & 0.18 & 0.18 \\
\hline \multirow{3}{*}{2} & VIC,QLD|NSW & Frank & 1.12 & $(0.15)$ & & & 0.12 & & \\
\hline & SA,NSW|VIC & Frank & 0.52 & $(0.15)$ & & & 0.06 & & \\
\hline & TAS,SA|VIC & Gumbel & 1.03 & $(0.01)$ & & & 0.03 & 0.04 & \\
\hline \multirow{2}{*}{3} & SA,QLD|VIC,NSW & Independent & & & & & 0.00 & & \\
\hline & TAS,NSW|SA,VIC & Independent & & & & & 0.00 & & \\
\hline \multirow[t]{2}{*}{4} & TAS,QLD|SA,VIC,NSW & Frank & -0.42 & $(0.15)$ & & & -0.05 & & \\
\hline & Type: R-vine & LogLik: 16 & 3.57 & AIC: -33 & 63.14 & BIC: & -3297.02 & & \\
\hline
\end{tabular}


Table 7

Joint Probability in Upper Tails

\begin{tabular}{|c|c|c|c|c|c|c|c|c|c|c|}
\hline & & \multicolumn{3}{|c|}{ Empirical } & \multicolumn{3}{|c|}{ Vine Copula } & \multicolumn{3}{|c|}{ Multivariate Student's $t$ Copula } \\
\hline & & Period 1 & Period 2 & Period 3 & Period 1 & Period 2 & Period 3 & Period 1 & Period 2 & Period 3 \\
\hline \multirow{4}{*}{$>0.90$} & at least 2 & $12.80 \%$ & $11.51 \%$ & $12.54 \%$ & $13.02 \%$ & $12.40 \%$ & $12.26 \%$ & $12.71 \%$ & $12.60 \%$ & $12.53 \%$ \\
\hline & at least 3 & $5.75 \%$ & $3.97 \%$ & $5.04 \%$ & $5.79 \%$ & $5.05 \%$ & $5.53 \%$ & $5.93 \%$ & $5.54 \%$ & $5.41 \%$ \\
\hline & at least 4 & $2.26 \%$ & $0.96 \%$ & $2.30 \%$ & $2.32 \%$ & $1.54 \%$ & $2.31 \%$ & $2.50 \%$ & $2.18 \%$ & $2.10 \%$ \\
\hline & 5 together & $0.82 \%$ & $0.14 \%$ & $0.44 \%$ & $0.70 \%$ & $0.22 \%$ & $0.58 \%$ & $0.75 \%$ & $0.60 \%$ & $0.56 \%$ \\
\hline \multirow{4}{*}{$>0.95$} & at least 2 & $5.54 \%$ & $3.29 \%$ & $5.42 \%$ & $6.23 \%$ & $5.22 \%$ & $5.65 \%$ & $5.89 \%$ & $5.73 \%$ & $5.73 \%$ \\
\hline & at least 3 & $1.78 \%$ & $1.23 \%$ & $2.03 \%$ & $2.15 \%$ & $1.76 \%$ & $2.29 \%$ & $2.46 \%$ & $2.25 \%$ & $2.22 \%$ \\
\hline & at least 4 & $0.62 \%$ & $0.41 \%$ & $0.44 \%$ & $0.69 \%$ & $0.37 \%$ & $0.89 \%$ & $0.95 \%$ & $0.81 \%$ & $0.78 \%$ \\
\hline & 5 together & $0.21 \%$ & $0.14 \%$ & $0.05 \%$ & $0.18 \%$ & $0.03 \%$ & $0.21 \%$ & $0.27 \%$ & $0.20 \%$ & $0.19 \%$ \\
\hline \multirow{4}{*}{$>0.99$} & at least 2 & $0.96 \%$ & $0.55 \%$ & $0.55 \%$ & $1.15 \%$ & $0.73 \%$ & $1.01 \%$ & $1.02 \%$ & $0.96 \%$ & $0.96 \%$ \\
\hline & at least 3 & $0.14 \%$ & $0.14 \%$ & $0.11 \%$ & $0.21 \%$ & $0.19 \%$ & $0.35 \%$ & $0.36 \%$ & $0.32 \%$ & $0.31 \%$ \\
\hline & at least 4 & $0.14 \%$ & $0.00 \%$ & $0.05 \%$ & $0.04 \%$ & $0.01 \%$ & $0.13 \%$ & $0.13 \%$ & $0.10 \%$ & $0.09 \%$ \\
\hline & 5 together & $0.00 \%$ & $0.00 \%$ & $0.00 \%$ & $0.01 \%$ & $0.00 \%$ & $0.03 \%$ & $0.04 \%$ & $0.02 \%$ & $0.02 \%$ \\
\hline
\end{tabular}

